\title{
An Efficient One-pot Synthesis of Unsymmetrical Ethers: A Directly Reductive Deoxygenation of Esters Using an $\mathrm{InBr}_{3} / \mathrm{Et}_{3} \mathrm{SiH}$ Catalytic System
}

\section{Supporting Information}

\author{
Norio Sakai,* Toshimitsu Moriya, and Takeo Konakahara
}

Department of Pure and Applied Chemistry, Faculty of Science and Technology, Tokyo University of Science (RIKADAI), Noda, Chiba 278-8510, Japan

E-mail: sakachem@rs.noda.tus.ac.jp

\section{Table of Contents}

S1-S7: $\quad$ Detailed procedure and Spectral Data for compounds prepared

S8-S29: $\quad$ Copies of ${ }^{1} \mathrm{H}$ NMR $/{ }^{13} \mathrm{C}$ NMR spectra for compounds prepared 


\section{Experimental Section}

General Methods: Column chromatography was performed using Silica gel 60. Chloroform and THF were distilled from $\mathrm{P}_{2} \mathrm{O}_{5}$ and sodium-benzophenone, respectively. Other organic solvents were dried and distilled prior to use. $\mathrm{InBr}_{3}, \mathrm{InCl}_{3}$, and $\mathrm{In}(\mathrm{OTf})_{3}$ were commercial available, and used without a further purification. All reactions were carried out under a nitrogen atmosphere, unless otherwise noted. ${ }^{1} \mathrm{H}$-NMR spectra were measured at $500 \mathrm{MHz}$ using tetramethylsilane as an internal standard. ${ }^{13} \mathrm{C}-\mathrm{NMR}$ spectra were measured at $125 \mathrm{MHz}$ using the center peak of chloroform (77.0 ppm) as an internal standard. High-resolution mass was measured using NBA (3-nitrobenzylalcohol) as a matrix. The ester compounds $\mathbf{1}$ and 3a-c, and $\mathbf{5 a}, \mathbf{b}$, which were prepared from the corresponding alcohol/thiol and acetyl chloride, are known compounds, and were identified by ${ }^{1} \mathrm{H}$ NMR and ${ }^{13} \mathrm{C}$ NMR by comparison to literature data. 2-Phenylethyl acetate (1a), benzyl acetate (1b), ethyl benzoate (1i), 3-isochromanone (1o), ethyl thiophenyl-2-acetate (1m), 3-phenylpropanoic acid (5c), and 4-phenyl-2-butanone (5d) were commercial available and used without a further purification. Phenethyl butyrate (1e), phenethyl isobutyrate (1f), and $\gamma$-lactone (1n) were also purchased.

General Procedure for the preparation of esters: An alcohol (10 mmol), acetyl chloride (20 mmol), and $\mathrm{Et}_{3} \mathrm{~N}(20 \mathrm{mmol})$ were successively added via a syringe to anhydrous $\mathrm{Et}_{2} \mathrm{O}(20 \mathrm{~mL})$ at room temperature under a $\mathrm{N}_{2}$ atmosphere. The reaction mixture was stirred for $2 \mathrm{~h}$. The resulting precipitation was filtered, and the filtrate was washed with $\mathrm{H}_{2} \mathrm{O}$. The organic layer was dried over anhydrous $\mathrm{Na}_{2} \mathrm{SO}_{4}$, and evaporated under reduced pressure. The crude product was purified by a silica gel chromatography (hexane : AcOEt $=8: 2$ ) to give the corresponding ester as shown in Table 2, Scheme 1, and Scheme 2.

4-Nitrophenethyl acetate $^{1)}$ (1c): 89\% yield; colorless needles; ${ }^{1} \mathrm{H}$ NMR $\left(500 \mathrm{MHz}, \mathrm{CDCl}_{3}\right) \delta 2.01(\mathrm{~s}$, $3 \mathrm{H}), 3.03(\mathrm{t}, 2 \mathrm{H}, J=6.5 \mathrm{~Hz}), 4.30(\mathrm{t}, 2 \mathrm{H}, J=6.5 \mathrm{~Hz}), 7.36(\mathrm{~d}, 2 \mathrm{H}, J=8.5 \mathrm{~Hz}), 8.14(\mathrm{~d}, 2 \mathrm{H}, J=8.5$ $\mathrm{Hz}) ;{ }^{13} \mathrm{C} \mathrm{NMR}\left(125 \mathrm{MHz}, \mathrm{CDCl}_{3}\right) \delta$ 20.8, 34.9, 63.8, 123.7, 129.7, 145.7, 146.9, 170.8; MS (EI) $\mathrm{m} / \mathrm{z}$ $209\left(\mathrm{M}^{+}\right), 151(100 \%)$.

3-Phenylpropyl acetate ${ }^{2}$ (1d): 99\% yield; yellow oil; ${ }^{1} \mathrm{H} \mathrm{NMR}\left(500 \mathrm{MHz}, \mathrm{CDCl}_{3}\right) \delta 1.97$ (quint, $2 \mathrm{H}, J$ $=7.0 \mathrm{~Hz}), 2.05(\mathrm{~s}, 3 \mathrm{H}), 2.69(\mathrm{t}, 2 \mathrm{H}, J=7.0 \mathrm{~Hz}), 4.09(\mathrm{t}, 2 \mathrm{H}, J=7.0), 7.18-7.30(\mathrm{~m}, 5 \mathrm{H}) ;{ }^{13} \mathrm{C} \mathrm{NMR}(125$ $\left.\mathrm{MHz}, \mathrm{CDCl}_{3}\right) \delta 20.9,30.2,32.2,63.8,126.0,128.3,128.4,141.2,171.1 ; \mathrm{MS}(\mathrm{EI}): m / z 178\left(\mathrm{M}^{+}\right), 91$ $(100 \%)$.

Methyl (4-bromophenyl)acetate ${ }^{3)}$ (1h): 89\% yield; colorless oil; ${ }^{1} \mathrm{H}$ NMR $\left(500 \mathrm{MHz}, \mathrm{CDCl}_{3}\right) \delta 3.58$ $(\mathrm{s}, 2 \mathrm{H}), 3.69$ (s, 3H), $7.15(\mathrm{~d}, 2 \mathrm{H}, J=8.5 \mathrm{~Hz}), 7.44(\mathrm{~d}, 2 \mathrm{H}, J=8.5 \mathrm{~Hz}) ;{ }^{13} \mathrm{C} \mathrm{NMR}\left(125 \mathrm{MHz}, \mathrm{CDCl}_{3}\right) \delta$ 40.5, 52.1, 121.2, 130.9, 131.4, 132.9, 171.4; MS (EI) $m / z .229,231\left(\mathrm{M}^{+}, \mathrm{M}^{+}+2\right)$.

n-Decyl acetate ${ }^{4)}$ (1j): 99\% yield; yellow oil; ${ }^{1} \mathrm{H}$ NMR $\left(500 \mathrm{MHz}, \mathrm{CDCl}_{3}\right) \delta 0.85(\mathrm{t}, 3 \mathrm{H}, J=7.0 \mathrm{~Hz})$, 
1.23-1.40 (m, 14H), 1.58 (quint, 2H, $J=7.0 \mathrm{~Hz}), 2.01(\mathrm{~s}, 3 \mathrm{H}), 4.02(\mathrm{t}, 2 \mathrm{H}, J=7.0 \mathrm{~Hz}) ;{ }^{13} \mathrm{C}$ NMR $(125$ $\mathrm{MHz}, \mathrm{CDCl}_{3}$ ) $\delta 14.0,20.9,22.6,25.9,28.6,29.2,29.3,29.5$ (d), 31.8, 64.6, 171.2; MS (FAB): $\mathrm{m} / z 201$ $(\mathrm{M}+\mathrm{H}, 100 \%)$.

1-Acetoxy-10-bromo decane ${ }^{5}$ (1k): 99\% yield; colorless oil; ${ }^{1} \mathrm{H}$ NMR $\left(500 \mathrm{MHz}, \mathrm{CDCl}_{3}\right) \delta$ 1.20-1.30 (m, 10H), $1.35(\mathrm{~m}, 2 \mathrm{H}), 1.55$ (t, 2H, $J=7.0 \mathrm{~Hz}$ ), 1.78 (quint, $2 \mathrm{H}, J=7.0 \mathrm{~Hz}$ ), $1.98(\mathrm{~s}, 3 \mathrm{H}$ ), 3.33 (t, 2H, $J=7.0 \mathrm{~Hz}), 3.98(\mathrm{t}, 2 \mathrm{H}, J=7.0 \mathrm{~Hz}) ;{ }^{13} \mathrm{C} \mathrm{NMR}\left(125 \mathrm{MHz}, \mathrm{CDCl}_{3}\right) \delta$ 21.0, 25.8, 28.1, 28.5, 28.7, 29.1, 29.2, 29.3, 32.8, 33.9, 64.5, 171.1; MS (EI) $m / z 278\left(\mathrm{M}^{+}\right), 280\left(\mathrm{M}^{+}+2\right), 107(100 \%)$.

2-Decyl acetate ${ }^{5)}$ (11): 99\% yield; colorless oil; ${ }^{1} \mathrm{H}$ NMR (500 MHz, $\left.\mathrm{CDCl}_{3}\right) \delta 0.88$ (t, 3H, $J=7.0 \mathrm{H}$ ), $1.21(\mathrm{t}, 3 \mathrm{H}, J=6.5 \mathrm{~Hz}), 1.26-1.30(\mathrm{~m}, 12 \mathrm{H}), 1.46(\mathrm{~m}, 1 \mathrm{H}), 1.58(\mathrm{~m}, 1 \mathrm{H}), 2.03(\mathrm{~s}, 3 \mathrm{H}), 4.89(\mathrm{sex}, 1 \mathrm{H}, J$ $=6.5 \mathrm{~Hz}) ;{ }^{13} \mathrm{C} \mathrm{NMR}\left(125 \mathrm{MHz}, \mathrm{CDCl}_{3}\right) \delta 14.1,19.9,21.4,22.6,25.4,29.2,29.4,29.5,31.8,35.9,71.1$, 170.8; MS (EI): $m / z 200\left(\mathrm{M}^{+}\right), 103(100 \%)$.

Diphenylmethyl acetate ${ }^{6}$ (3a): $88 \%$ yield; yellow oil; ${ }^{1} \mathrm{H}$ NMR $\left(500 \mathrm{MHz}, \mathrm{CDCl}_{3}\right) \delta 2.16(\mathrm{~s}, 3 \mathrm{H})$,

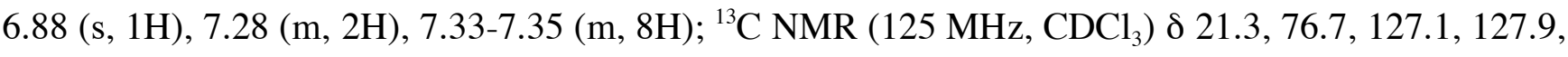
128.5, 140.2, 170.0; MS (EI): $\mathrm{m} / z 226\left(\mathrm{M}^{+}\right), 166(100 \%)$.

$\boldsymbol{\alpha}$-Methylbenzyl acetate ${ }^{6}$ (3b): 92\% yield; yellow oil; ${ }^{1} \mathrm{H}$ NMR $\left(500 \mathrm{MHz}, \mathrm{CDCl}_{3}\right) \delta 1.53(\mathrm{~d}, 3 \mathrm{H}, J=$ $6.5 \mathrm{~Hz}), 2.07$ (s, 3H), 5.89 (q, 1H, $J=6.5 \mathrm{~Hz}), 7.29(\mathrm{~m}, 1 \mathrm{H}), 7.33(\mathrm{~m}, 4 \mathrm{H}) ;{ }^{13} \mathrm{C}$ NMR $(125 \mathrm{MHz}$, $\left.\mathrm{CDCl}_{3}\right) \delta$ 21.3, 22.2, 72.3, 126.1, 127.8, 128.5, 141.7, 170.3; MS (EI): $\mathrm{m} / z 164\left(\mathrm{M}^{+}\right), 104$ (100\%).

4-Phenyl-3-butene-2-ol acetate ${ }^{7)}$ (3c): 99\% yield; colorless oil; ${ }^{1} \mathrm{H}$ NMR $\left(500 \mathrm{MHz}, \mathrm{CDCl}_{3}\right) \delta 1.33$ (d, $3 \mathrm{H}, J=6.5 \mathrm{~Hz}$ ), 2.00 (s, 3H), 5.45 (quint, $1 \mathrm{H}, J=6.5 \mathrm{~Hz}$ ), 6.12 (dd, $1 \mathrm{H}, J=16.0,7.5 \mathrm{~Hz}), 6.53$ (d, 1H, $J=16.0 \mathrm{~Hz}), 7.17(\mathrm{~m}, 1 \mathrm{H}), 7.25(\mathrm{~m}, 2 \mathrm{H}), 7.30(\mathrm{~m}, 2 \mathrm{H}) ;{ }^{13} \mathrm{C} \mathrm{NMR}\left(125 \mathrm{MHz}, \mathrm{CDCl}_{3}\right) \delta 20.4,21.4,71.0$, 126.5, 127.9, 128.5, 128.8, 131.5, 136.3, 170.3; MS (EI) $\mathrm{m} / z 190\left(\mathrm{M}^{+}\right), 129$ (100\%).

Phenethyl thioacetate $^{8)}$ (5a): 86\% yield; colorless oil; ${ }^{1} \mathrm{H}$ NMR (500 MHz, $\mathrm{CDCl}_{3}$ ) $\delta 2.33(\mathrm{~s}, 3 \mathrm{H}), 2.87$ $(\mathrm{t}, 2 \mathrm{H}, J=7.5 \mathrm{~Hz}), 3.12(\mathrm{t}, 2 \mathrm{H}, J=7.5 \mathrm{~Hz}), 7.23(\mathrm{~m}, 3 \mathrm{H}), 7.30(\mathrm{~m}, 2 \mathrm{H}) ;{ }^{13} \mathrm{C}$ NMR $\left(125 \mathrm{MHz}, \mathrm{CDCl}_{3}\right) \delta$ 30.5, 30.7, 35.8, 126.5, 128.5, 128.6, 140.0, 195.7; MS (EI): $\mathrm{m} / \mathrm{z} 180\left(\mathrm{M}^{+}\right), 75$ (100\%).

$N$-Phenethylacetamide) (5b): 91\% yield; colorless oil; ${ }^{1} \mathrm{H}$ NMR (500 MHz, $\left.\mathrm{CDCl}_{3}\right) \delta 1.90$ (s, 3H), $2.80(\mathrm{t}, 2 \mathrm{H}, J=7.0 \mathrm{~Hz}), 3.48(\mathrm{~d}, 2 \mathrm{H}, J=7.0 \mathrm{~Hz}), 7.17-7.23(\mathrm{~m}, 3 \mathrm{H}), 7.27-7.31(\mathrm{~m}, 2 \mathrm{H}) ;{ }^{13} \mathrm{C} \mathrm{NMR}(125$ $\left.\mathrm{MHz}, \mathrm{CDCl}_{3}\right) \delta 23.1,35.5,40.6,126.3,128.5,128.6,138.8,170.1 ; \mathrm{MS}(\mathrm{EI}): \mathrm{m} / z 163\left(\mathrm{M}^{+}\right)$.

General procedure for the reduction of esters using the $\mathbf{I n B r}_{3}-\mathbf{E t}_{3} \mathbf{S i H}$ system: To a freshly distilled $\mathrm{CHCl}_{3}$ solution $(0.6 \mathrm{~mL})$ in a screw-capped vial under $\mathrm{N}_{2}$ atmosphere, ester $1(0.6 \mathrm{mmol}), \operatorname{InBr}_{3}(10.6$ $\mathrm{mg}, 0.0300 \mathrm{mmol})$ and $\mathrm{Et}_{3} \mathrm{SiH}(380 \mathrm{~mL}, 2.40 \mathrm{mmol})$ was successively added, and the vial was sealed with a cap containing a PTFE septum. During the stirring of the reaction mixture at $60{ }^{\circ} \mathrm{C}$ (bath temperature), the solution turned from colorless to yellow, then to orange. The reaction was monitored 
by $\mathrm{GC}$ analysis until consumption of the starting ester. After the reaction, $\mathrm{H}_{2} \mathrm{O}(3 \mathrm{~mL})$ was added, and the resulting orange suspension was stirred continuously until the disappearance of the color. The aqueous layer was extracted with $\mathrm{CH}_{2} \mathrm{Cl}_{2}(15 \mathrm{~mL})$, the combined organic phase was dried over anhydrous $\mathrm{Na}_{2} \mathrm{SO}_{4}$ filtered, and then evaporated under reduced pressure. The crude product was purified by flash column chromatography $\left(\mathrm{SiO}_{2} /\right.$ hexane $:$ AcOEt $\left.=99: 1\right)$ to give the corresponding ether 2 as shown in Table 2, and in Scheme 2.

Ethyl phenethyl ether ${ }^{10)}$ (2a): 86\% yield; colorless oil; ${ }^{1} \mathrm{H}$ NMR (500 MHz, $\left.\mathrm{CDCl}_{3}\right) \delta 1.20(\mathrm{t}, 3 \mathrm{H}, J=7.5 \mathrm{~Hz}), 2.90(\mathrm{t}, 2 \mathrm{H}, J=7.5 \mathrm{~Hz}), 3.50(\mathrm{q}, 2 \mathrm{H}, J=7.5 \mathrm{~Hz})$, $3.63(\mathrm{t}, 2 \mathrm{H}, J=7.5 \mathrm{~Hz}), 7.22(\mathrm{~m}, 3 \mathrm{H}), 7.28(\mathrm{~m}, 2 \mathrm{H}) ;{ }^{13} \mathrm{C} \mathrm{NMR}\left(125 \mathrm{MHz}, \mathrm{CDCl}_{3}\right)$

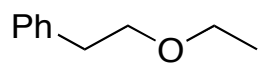

$\delta$ 15.2, 36.4, 66.2, 71.6, 126.1, 128.3, 128.9, 139.0; MS (EI): $m / z .150\left(\mathrm{M}^{+}\right), 105$ $(100 \%)$.

Ethyl benzyl ether ${ }^{11)}$ (2b): 69\% yield; colorless oil; ${ }^{1} \mathrm{H}$ NMR (500 MHz, $\left.\mathrm{CDCl}_{3}\right) \delta$ $1.25(\mathrm{t}, 3 \mathrm{H}, J=7.0 \mathrm{~Hz}), 3.54(\mathrm{q}, 2 \mathrm{H}, J=7.0 \mathrm{~Hz}), 4.51(\mathrm{~s}, 2 \mathrm{H}), 7.26(\mathrm{~m}, 1 \mathrm{H}), 7.33(\mathrm{~m}$, $\mathrm{Ph}_{\mathrm{O}} \widehat{\mathrm{C}}$ $4 \mathrm{H}) ;{ }^{13} \mathrm{C} \mathrm{NMR}\left(125 \mathrm{MHz}, \mathrm{CDCl}_{3}\right) \delta 15.2,65.7,72.7,127.5,127.7,128.3,138.6$; MS (EI): $m / z 136\left(\mathrm{M}^{+}\right), 91(100 \%)$.

Ethyl 4-nitrophenethyl ether (2c): $61 \%$ yield; brown oil; ${ }^{1} \mathrm{H}$ NMR (500 $\left.\mathrm{MHz} \mathrm{CDCl}_{3}\right) \delta 1.19(\mathrm{t}, 3 \mathrm{H}, J=7.0 \mathrm{~Hz}), 2.98(\mathrm{t}, 2 \mathrm{H}, J=7.0 \mathrm{~Hz}), 3.49(\mathrm{t}$, $2 \mathrm{H}, J=7.0 \mathrm{~Hz}), 3.67(\mathrm{t}, 2 \mathrm{H}, J=7.0 \mathrm{~Hz}), 7.39$ (d, 2H, $J=8.5 \mathrm{~Hz}), 8.15(\mathrm{~d}$, $2 \mathrm{H}, J=8.5 \mathrm{~Hz}) ;{ }^{13} \mathrm{C} \mathrm{NMR}\left(125 \mathrm{MHz}, \mathrm{CDCl}_{3}\right) \delta 15.1,36.2,66.4,70.3$,

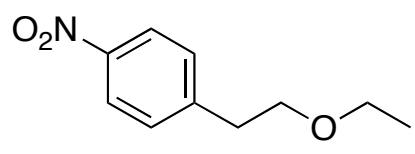
123.5, 129.7, 146.6, 147.3; MS (FAB): $m / z 196\left(\mathrm{M}^{+}+1,100 \%\right)$, HRMS (FAB): Calcd for $\mathrm{C}_{10} \mathrm{H}_{14} \mathrm{NO}_{3}$ : 196.0974, Found: 196.0978.

Ethyl 3-phenylpropyl ether ${ }^{12)}$ (2d): 89\% yield; colorless oil; ${ }^{1} \mathrm{H}$ NMR (500 MHz, $\left.\mathrm{CDCl}_{3}\right) \delta 1.21(\mathrm{t}, 3 \mathrm{H}, J=7.0 \mathrm{~Hz}), 1.90(\mathrm{~m}, 2 \mathrm{H}), 2.69(\mathrm{t}, 2 \mathrm{H}, J=7.0 \mathrm{~Hz}), 3.42(\mathrm{t}$, $2 \mathrm{H}, J=7.0 \mathrm{~Hz}), 3.46(\mathrm{q}, 2 \mathrm{H}, J=7.0 \mathrm{~Hz}), 7.17(\mathrm{~m}, 3 \mathrm{H}), 7.27(\mathrm{~m}, 2 \mathrm{H}) ;{ }^{13} \mathrm{C} \mathrm{NMR}$

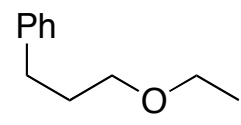
$\left(125 \mathrm{MHz} \mathrm{CDCl}_{3}\right) \delta 15.2,31.3,32.4,66.1,69.7,125.7,128.3,128.4,142.0 ; \mathrm{MS}$ (EI): $m / z 164\left(\mathrm{M}^{+}\right), 118(100 \%)$.

n-Butyl phenethyl ether ${ }^{13)}$ (2e): $71 \%$ yield; colorless oil; ${ }^{1} \mathrm{H}$ NMR (500 MHz, $\left.\mathrm{CDCl}_{3}\right) \delta 0.91(\mathrm{t}, 3 \mathrm{H}, J=7.0 \mathrm{~Hz}), 1.35(\mathrm{sex}, 2 \mathrm{H}, J=7.0 \mathrm{~Hz}), 1.56$ (quint, $2 \mathrm{H}, J$ $=7.0 \mathrm{~Hz}), 2.89(\mathrm{t}, 2 \mathrm{H}, J=7.0 \mathrm{~Hz}), 3.43(\mathrm{t}, 2 \mathrm{H}, J=7.0 \mathrm{~Hz}), 3.62(\mathrm{t}, 2 \mathrm{H}, J=7.0$ $\mathrm{Ph} \sim \sim_{\mathrm{O}}$ $\mathrm{Hz}), 7.20(\mathrm{~m}, 3 \mathrm{H}), 7.28(\mathrm{~m}, 2 \mathrm{H}) ;{ }^{13} \mathrm{C} \mathrm{NMR}\left(125 \mathrm{MHz}, \mathrm{CDCl}_{3}\right) \delta 13.9,19.3$, 31.8, 36.4, 70.8, 71.8, 126.1, 128.3, 128.9, 139.1; MS (EI): m/z $178\left(\mathrm{M}^{+}\right), 57$ $(100 \%)$. 
Phenethyl isobutyl ether (2f): $71 \%$ yield; colorless oil; ${ }^{1} \mathrm{H}$ NMR $(500 \mathrm{MHz}$, $\left.\mathrm{CDCl}_{3}\right) \delta 0.89(\mathrm{~d}, 6 \mathrm{H}, J=7.0 \mathrm{~Hz}), 1.84(\mathrm{~m}, 1 \mathrm{H}), 2.89(\mathrm{t}, 2 \mathrm{H}, J=7.0 \mathrm{~Hz}), 3.20(\mathrm{~d}$, $2 \mathrm{H}, J=6.5 \mathrm{~Hz}), 3.62(\mathrm{t}, 2 \mathrm{H}, J=7.0 \mathrm{~Hz}), 7.20(\mathrm{~m}, 3 \mathrm{H}), 7.28(\mathrm{~m}, 2 \mathrm{H}) ;{ }^{13} \mathrm{C} \mathrm{NMR}$ $\left(125 \mathrm{MHz}, \mathrm{CDCl}_{3}\right) \delta 19.4,28.4,36.4,71.9,76.7,126.1,128.3,128.9,139.2 ; \mathrm{MS}$

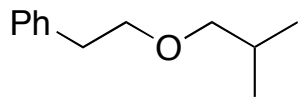
(FAB): $m / z 178\left(\mathrm{M}^{+}\right), 105$ (100\%); HRMS (FAB) Calcd for $\mathrm{C}_{12} \mathrm{H}_{18} \mathrm{O}$ 178.1358, Found 178.1337.

Methyl 4-bromophenethyl ether $^{14)}$ (2h): $62 \%$ yield; colorless oil; ${ }^{1} \mathrm{H}$ NMR $\left(500 \mathrm{MHz}, \mathrm{CDCl}_{3}\right) \delta 2.83(\mathrm{t}, 2 \mathrm{H}, J=7.0 \mathrm{~Hz}), 3.34(\mathrm{~s}, 3 \mathrm{H}), 3.57(\mathrm{t}, 2 \mathrm{H}, J=7.0$ $\mathrm{Hz}$ ), 7.09 (d, 2H, J = 8.0 Hz), 7.40 (d, 2H, J = 8.0 Hz); ${ }^{13} \mathrm{C}$ NMR (125 MHz, $\left.\mathrm{CDCl}_{3}\right) \delta 35.6,58.7,73.1,120.0,130.9,131.4,138.0$; MS (EI) $m / z$ 215, 217

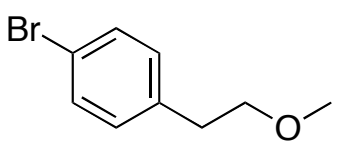
$\left(\mathrm{M}^{+}, \mathrm{M}^{+}+2\right)$.

1-Ethoxydecane ${ }^{10)}$ (2j): $77 \%$ yield; colorless oil; ${ }^{1} \mathrm{H}$ NMR $\left(500 \mathrm{MHz}, \mathrm{CDCl}_{3}\right) \delta$ $0.88(\mathrm{t}, 3 \mathrm{H}, J=7.0 \mathrm{~Hz}), 1.20(\mathrm{t}, 3 \mathrm{H}, J=7.0 \mathrm{~Hz}), 1.25-1.40(\mathrm{~m}, 14 \mathrm{H}), 1.57$ (quint, $2 \mathrm{H}, J=7.0 \mathrm{~Hz}), 3.40(\mathrm{t}, 2 \mathrm{H}, J=7.0 \mathrm{~Hz}), 3.46(\mathrm{q}, 2 \mathrm{H}, J=7.0 \mathrm{~Hz}) ;{ }^{13} \mathrm{C}$ NMR $(125$ $\left.\mathrm{MHz}_{\mathrm{CDCl}}\right) \delta 14.1,15.2,22.7,26.2,29.3,29.5,29.7,29.6,29.8,31.9,66.0$, 70.8; MS (EI) $m / z 186\left(\mathrm{M}^{+}, 100 \%\right)$.

1-Bromo-10-ethoxydecane ${ }^{15)}$ (2k): $73 \%$ yield; colorless oil; ${ }^{1} \mathrm{H}$ NMR (500 $\left.\mathrm{MHz} \mathrm{CDCl}_{3}\right) \delta 1.20(\mathrm{t}, 3 \mathrm{H}, J=7.5 \mathrm{~Hz}), 1.25-1.30(\mathrm{~m}, 10 \mathrm{H}), 1.42(\mathrm{~m}, 2 \mathrm{H})$, $1.56(\mathrm{t}, 2 \mathrm{H}, J=7.5 \mathrm{~Hz}), 1.85$ (quint, $2 \mathrm{H}, J=7.5 \mathrm{~Hz}$ ), $3.40(\mathrm{t}, 4 \mathrm{H}, J=7.5 \mathrm{~Hz}$ ), 3.46 (q, 2H, $J=7.5 \mathrm{~Hz}) ;{ }^{13} \mathrm{C}$ NMR $\left(125 \mathrm{MHz}, \mathrm{CDCl}_{3}\right) \delta 15.1,26.1$, 28.0, 28.6,

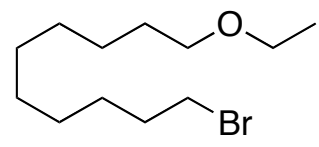
29.2, 29.3, 29.4, 29.7, 32.7, 33.7, 65.9, 70.6; MS (EI): m/z $264\left(\mathrm{M}^{+}\right), 266$ $\left(\mathrm{M}^{+}+2\right), 71(100 \%)$.

2-Ethoxydecane ${ }^{5)}$ (2l): $69 \%$ yield; colorless oil; ${ }^{1} \mathrm{H}$ NMR $\left(500 \mathrm{MHz}, \mathrm{CDCl}_{3}\right) \delta$ $0.81(\mathrm{t}, 3 \mathrm{H}, J=7.0 \mathrm{~Hz}), 1.05(\mathrm{~d}, 3 \mathrm{H}, J=6.0 \mathrm{~Hz}), 1.12(\mathrm{t}, 3 \mathrm{H}, J=7.0 \mathrm{~Hz}), 1.20$ 1.25 (m, 11H), 1.28 (brs, 2H), 1.45 (brs, 1H), 3.29 (sex, 1H, J=6.0 Hz), 3.34 (quint, $1 \mathrm{H}, J=7.0 \mathrm{~Hz}$ ), 3.46 (quint, $1 \mathrm{H}, J=7.0 \mathrm{~Hz}$ ); ${ }^{13} \mathrm{C} \mathrm{NMR}(125 \mathrm{MHz}$, $\left.\mathrm{CDCl}_{3}\right) \delta 14.1,15.6,19.8,22.7,25.6,29.3,29.6,29.8,31.9,36.7,63.5,75.2$; MS (EI) $m / z, 186\left(\mathrm{M}^{+}\right), 73(100 \%)$.

1-Ethoxy-2-(2-thiophenyl)ethane (2m): 55\% yield; colorless oil; ${ }^{1} \mathrm{H}$ NMR (500 $\left.\mathrm{MHz} \mathrm{CDCl}_{3}\right) \delta 1.22(\mathrm{t}, 3 \mathrm{H}, J=7.0 \mathrm{~Hz}), 3.10(\mathrm{t}, 2 \mathrm{H}, J=7.0 \mathrm{~Hz}), 3.53(\mathrm{dd}, 2 \mathrm{H}, J$ $=7.0,7.0 \mathrm{~Hz}), 3.66(\mathrm{t}, 2 \mathrm{H}, \mathrm{J}=7.0 \mathrm{~Hz}), 6.86(\mathrm{~d}, 1 \mathrm{H}, J=3.5 \mathrm{~Hz}), 6.93(\mathrm{dd}, 1 \mathrm{H}, J=$ 5.5, 3.5 Hz), $7.14(\mathrm{~d}, 1 \mathrm{H}, J=5.5 \mathrm{~Hz}) ;{ }^{13} \mathrm{C} \mathrm{NMR}\left(125 \mathrm{MHz}, \mathrm{CDCl}_{3}\right) \delta$ 15.2, 30.5,

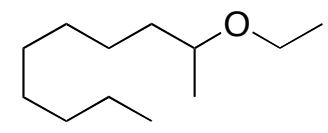
66.3, 71.2, 123.5, 125.0, 126.6, 141.3; MS (EI) $m / z 156\left(\mathrm{M}^{+}\right)$. HRMS (FAB): 
Calcd for $\mathrm{C}_{8} \mathrm{H}_{13} \mathrm{OS}: 157.0687$, Found: 157.0690 .

Isochroman ${ }^{16)}$ (2o): $84 \%$ yield; colorless oil; ${ }^{1} \mathrm{H}$ NMR $\left(500 \mathrm{MHz}, \mathrm{CDCl}_{3}\right) \delta 2.86(\mathrm{t}, 2 \mathrm{H}$, $J=5.5 \mathrm{~Hz}), 3.97(\mathrm{t}, 2 \mathrm{H}, J=5.5 \mathrm{~Hz}), 4.77(\mathrm{~s}, 2 \mathrm{H}), 6.97(\mathrm{~m}, 1 \mathrm{H}), 7.11(\mathrm{~m}, 1 \mathrm{H}), 7.16(\mathrm{~m}$, $2 \mathrm{H}) ;{ }^{13} \mathrm{C} \mathrm{NMR}\left(\mathrm{CDCl}_{3}\right) \delta$ 28.3, 65.4, 67.9, 124.4, 125.9, 126.3, 128.9, 133.2, 134.9; MS

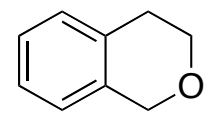
(EI) $\mathrm{m} / z 134\left(\mathrm{M}^{+}\right), 104(100 \%)$.

Diphenylmethane $^{17)}$ (4a): 98\% yield: colorless oil; ${ }^{1} \mathrm{H}$ NMR (500 MHz, $\left.\mathrm{CDCl}_{3}\right) \delta 3.98$ (s, 2H), $7.20(\mathrm{~m}, 6 \mathrm{H}), 7.27(\mathrm{~m}, 4 \mathrm{H}) ;{ }^{13} \mathrm{C} \mathrm{NMR}\left(125 \mathrm{MHz}, \mathrm{CDCl}_{3}\right) \delta 41.9,126.0,128.4$, $\mathrm{Ph} \widehat{\mathrm{Ph}}$ 128.9, 141.1; MS (EI) $\mathrm{m} / z 168\left(\mathrm{M}^{+}, 100 \%\right)$.

Ethylbenzene $^{17)}$ (4b): $76 \%$ yield; colorless oil; ${ }^{1} \mathrm{H}$ NMR (500 MHz, $\left.\mathrm{CDCl}_{3}\right) \delta 1.24$ (t, 3H, $J=7.5 \mathrm{~Hz}), 2.65$ (q, 2H, $J=7.5 \mathrm{~Hz}), 7.18(\mathrm{~m}, 3 \mathrm{H}), 7.28(\mathrm{~m}, 2 \mathrm{H}) ;{ }^{13} \mathrm{C} \mathrm{NMR}(125 \mathrm{MHz}$, $\mathrm{Ph} \widehat{\Upsilon}$ $\left.\mathrm{CDCl}_{3}\right) \delta 15.6,28.9,125.6,127.8,128.3,131.8,144.2$; MS (EI) $\mathrm{m} / z 106\left(\mathrm{M}^{+}\right), 91$ $(100 \%)$.

$\beta$-Ethyl styrene ${ }^{18)}$ (4c): $41 \%$ yield; colorless oil; ${ }^{1} \mathrm{H}$ NMR (500 MHz, $\left.\mathrm{CDCl}_{3}\right) \delta 1.09$ (t, $3 \mathrm{H}, J=7.5 \mathrm{~Hz}$ ), 2.23 (quint, $2 \mathrm{H}, J=7.5 \mathrm{~Hz}$ ), 6.27 (dt, $1 \mathrm{H}, J=6.5 \mathrm{~Hz}, 16 \mathrm{~Hz}$ ), 6.37 $(\mathrm{d}, 1 \mathrm{H}, J=16 \mathrm{~Hz}), 7.18(\mathrm{~m}, 2 \mathrm{H}), 7.27(\mathrm{~m}, 2 \mathrm{H}), 7.34(\mathrm{~m}, 1 \mathrm{H}) ;{ }^{13} \mathrm{C}$ NMR $(125 \mathrm{MHz}$, $\left.\mathrm{CDCl}_{3}\right) \delta$ 13.6, 26.1, 125.9, 126.7, 128.5, 128.8, 132.6, 137.9; MS (EI): $\mathrm{m} / z 132\left(\mathrm{M}^{+}\right)$. 1-Phenyl-2-butene ${ }^{19)}\left(\mathbf{4} \mathbf{c}^{\prime}\right): 18 \%$ yield; colorless oil; ${ }^{1} \mathrm{H}$ NMR $\left(500 \mathrm{MHz}, \mathrm{CDCl}_{3}\right) \delta$ $1.68(\mathrm{~d}, 3 \mathrm{H}, J=6.0 \mathrm{~Hz}), 3.31(\mathrm{~d}, 2 \mathrm{H}, J=6.5 \mathrm{~Hz}), 5,48-5.54(\mathrm{~m}, 1 \mathrm{H}), 5.56-5.62(\mathrm{~m}$, $\mathrm{Ph} \leadsto$ 1H), $7.18(\mathrm{~m}, 2 \mathrm{H}), 7.27(\mathrm{~m}, 2 \mathrm{H}), 7.34(\mathrm{~m}, 1 \mathrm{H}) ;{ }^{13} \mathrm{C} \mathrm{NMR}\left(125 \mathrm{MHz}, \mathrm{CDCl}_{3}\right) \delta$ 17.9, 39.0, 125.9, 126.3, 128.3, 128.4, 130.0, 141.1; MS (EI): $\mathrm{m} / z 132\left(\mathrm{M}^{+}\right)$.

3-Phenyl-1-propanol ${ }^{20)}$ (6c): $32 \%$ yield; colorless oil; ${ }^{1} \mathrm{H}$ NMR $(500 \mathrm{MHz}$, $\mathrm{CDCl}_{3}$ ) $\delta 1.45$ (brs, $1 \mathrm{H}$ ), 1.90 (quint, $2 \mathrm{H}, J=7.5 \mathrm{~Hz}$ ), 2.71 (t, $2 \mathrm{H}, J=7.5 \mathrm{~Hz}$ ), $3.67(\mathrm{t}, 2 \mathrm{H}, J=7.5 \mathrm{~Hz}), 7.20(\mathrm{~m}, 3 \mathrm{H}), 7.28(\mathrm{~m}, 2 \mathrm{H}) ;{ }^{13} \mathrm{C} \mathrm{NMR}\left(125 \mathrm{MHz}, \mathrm{CDCl}_{3}\right)$ $\delta$ 32.1, 34.2, 62.2, 125.8, 128.4, 141.8; MS (EI): $\mathrm{m} / z 136\left(\mathrm{M}^{+}\right)$.

Bis(4-phenyl-2-butyl)ether ${ }^{21)}$ (6d): $88 \%$ yield; colorless oil; ${ }^{1} \mathrm{H}$ NMR $(500 \mathrm{MHz}$, $\left.\mathrm{CDCl}_{3}\right) \delta 1.15(\mathrm{dd}, 3 \mathrm{H}, J=6,16 \mathrm{~Hz}), 1.72(\mathrm{~m}, 1 \mathrm{H}), 1.82(\mathrm{~m}, 1 \mathrm{H}), 2.63(\mathrm{~m}, 1 \mathrm{H})$, $2.73(\mathrm{~m}, 1 \mathrm{H}), 3.48(\mathrm{~m}, 1 \mathrm{H}), 7.17(\mathrm{~m}, 3 \mathrm{H}), 7.27(\mathrm{~m}, 2 \mathrm{H}) ;{ }^{13} \mathrm{C}$ NMR $(125 \mathrm{MHz}$, $\mathrm{CDCl}_{3}$ ) $\delta 20.7$ (d), 32.0 (d), 38.9 (d), 72.5 (d), 125.6 (d), 128.3 (d), 128.4 (d),<smiles>CC(CCc1ccccc1)OC(C)CCc1ccccc1</smiles>
142.4 (d); MS (FAB): m/z $283\left(\mathrm{M}^{+}+\mathrm{H}\right), 133$ (100\%); HRMS (FAB): Calcd for $\mathrm{C}_{20} \mathrm{H}_{27} \mathrm{O}: 283.2061$, Found: 283.2062 . 


\section{References}

1) Kawada, A.; Takeda, S.; Yamashita, K.; Abe, H.; Harayama, T. Chem. Pharm. Bull. 2002, 50, 1060 .

2) Davies, H. M. L.; Beckwith, R. E. J.; Antoulinakis, E. G.; Jin, Q. J. Org. Chem. 2003, 68, 6126.

3) Tamura, Y.; Yakura, T.; Shirouchi, Y.; Haruta, J.; Chem. Pharm. Bull. 1985, 33, 1097.

4) Nikolaides, N.; Ganem, B. J. Org. Chem. 1989, 54, 5996.

5) Gasparrini, F.; Caglioti, L.; Misiti, D.; Palmieri, G.; Ballini, R. Tetrahedron 1982, 38, 3609.

6) Karimi, B.; Maleki. J. J. Org. Chem. 2003, 68, 4591.

7) Onaran, M.; Seto, C. T. J. Org. Chem. 2003, 68, 8136.

8) Holmes, B. T.; Snow, A. W. Tetrahedron 2005, 61, 12339.

9) Kita, Y.; Akai, S.; Ajimura, N.; Yoshigi, M.; Tsugoshi, T.; Yasuda, H.; Tamura, Y. J. Org. Chem. 1986, 51, 4150 .

10) Oka, K.; Nakao, R. J. Organomet. Chem. 1989, 362, 31.

11) Ochiai, M.; Ito, T.; Takahashi, H.; Nakanishi, A.; Toyonari, M.; Sueda, T.; Goto, S.; Shiro, M. J. Am. Chem. Soc. 1996, 118, 7716.

12) (a) Ishikawa, H.; Mukaiyama, T. Bull. Chem. Soc. Jpn. 1978, 51, 2059. (b) Matsubara, K.; Iura, T.; Maki, T.; Nagashima, H. J. Org. Chem. 2002, 67, 4985.

13) Inoue, K.; Sawada, A.; Shibata, I.; Baba, A. J. Am. Chem. Soc. 2002, 124, 906.

14) Yato, M.; Homma, K.; Ishida, A. Tetrahedron 2001, 57, 5353.

15) Murayama, T.; Nagai, M.; Fujiyama, T.; Sakai, Y. JP Patent, 2000, 16969 (Chem. Abstr. 2000, 132, 100535).

16) Adcock, W.; Gupta, B. D.; Kitching, W. J. Org. Chem. 1976, 41, 1498.

17) Shapiro, M. J. J. Org. Chem. 1976, 41, 3197.

18) J. M. Concellón, ; H. Rodríguez-Solla; C. Simal; H. Huerta, H. Org. Lett. 2005, 7, 5833.

19) McEnroe, F. J.; Sha, C.-K.; Hall, S. S. J. Org. Chem. 1976, 41, 3465.

20) Chen, G.; Fu, C.; Ma, S. Tetrahedron 2006, 62, 4444.

21) Wada, M.; Nagayama, S.; Mizutani, K.; Hiroi, R.; Miyoshi, N. Chem. Lett. 2002, 31, 248. 

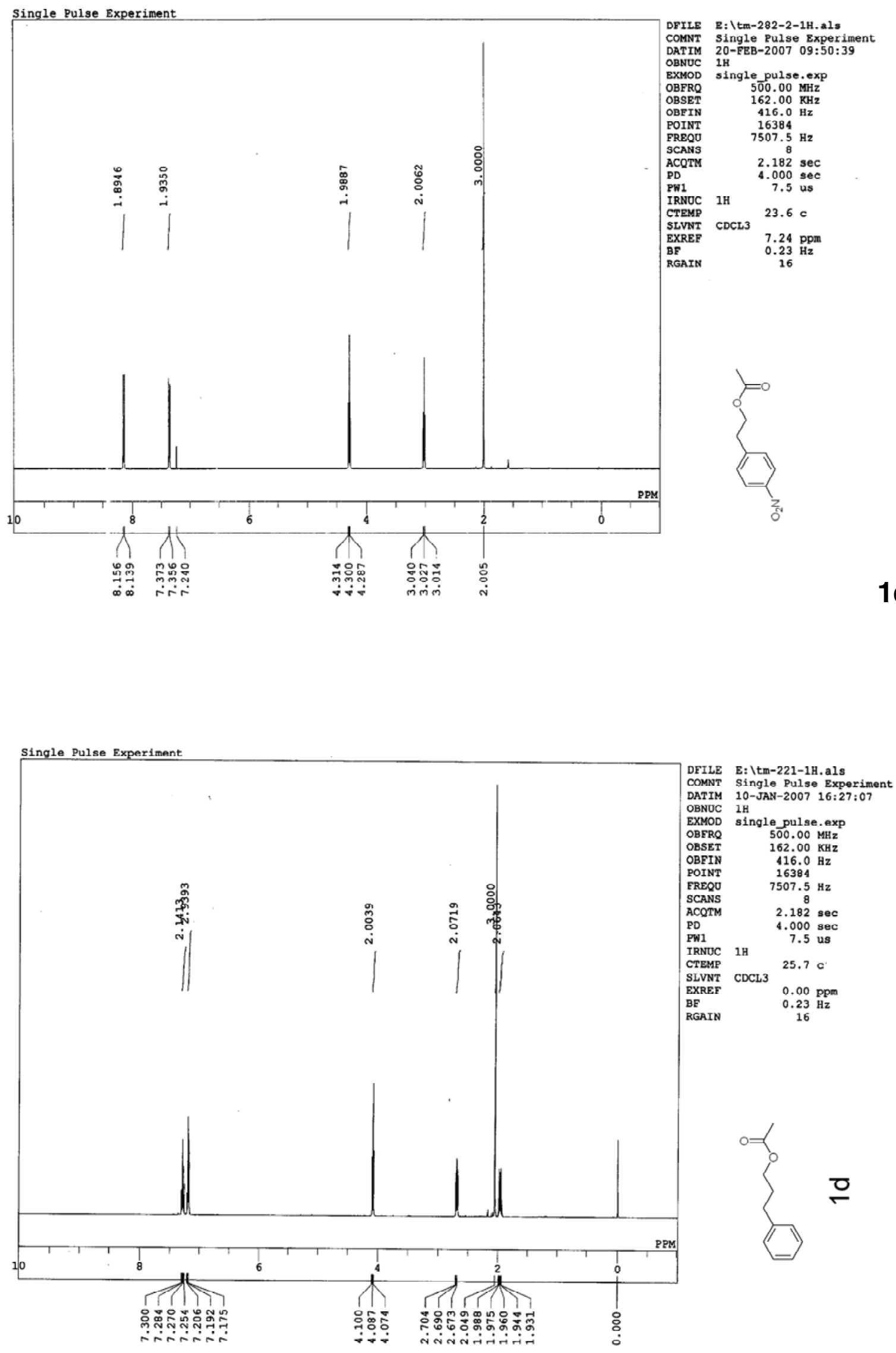


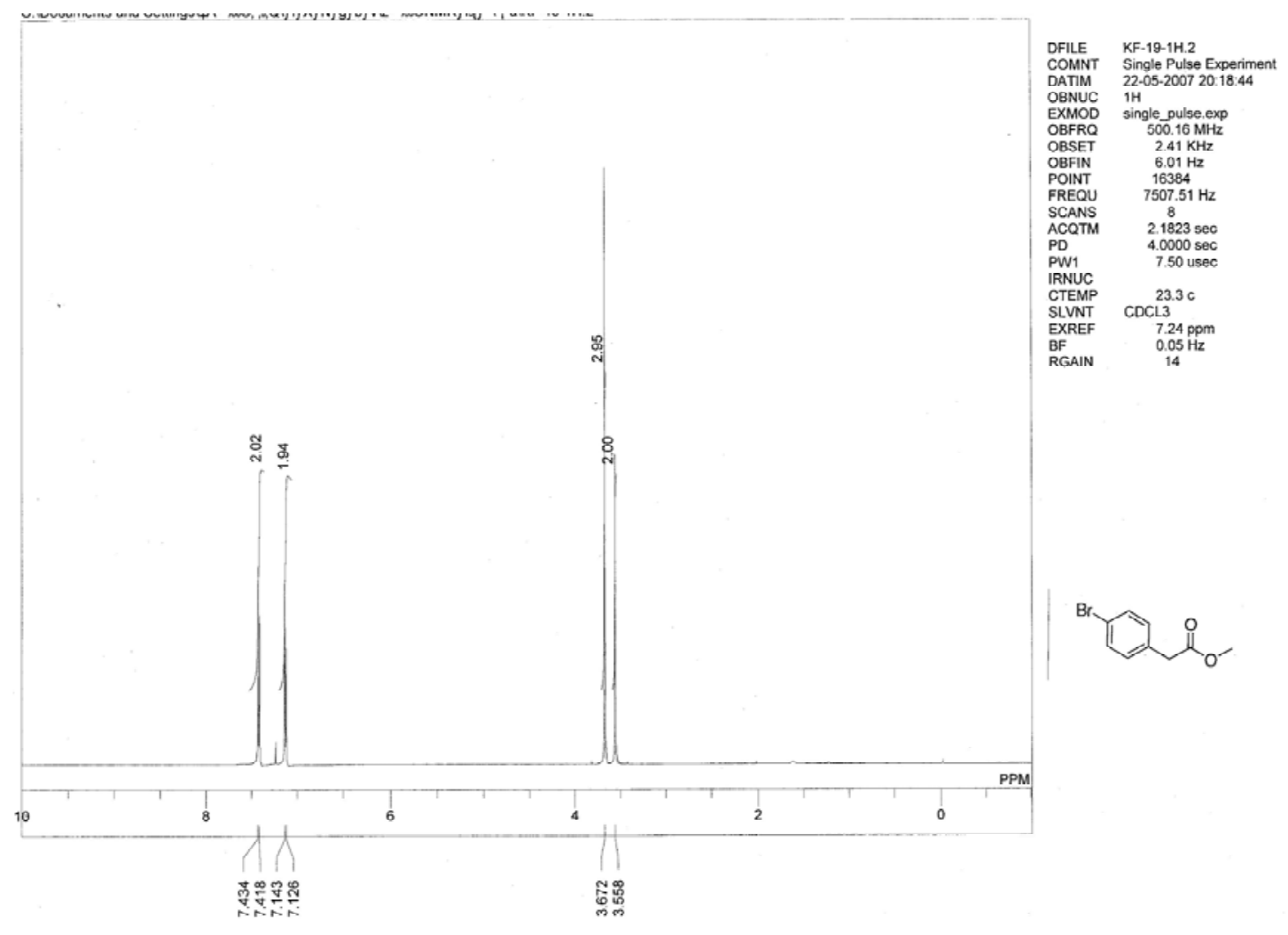

1h

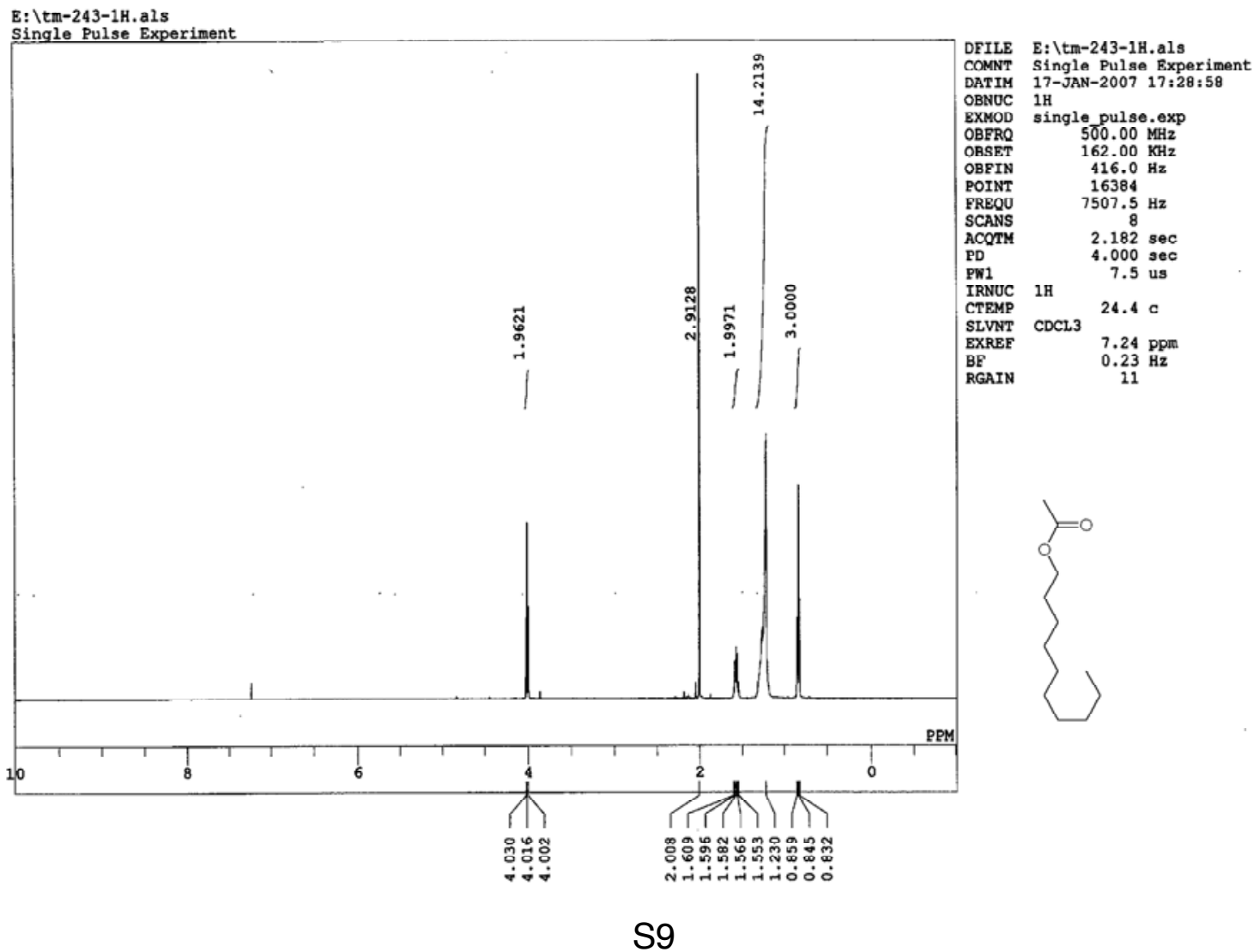




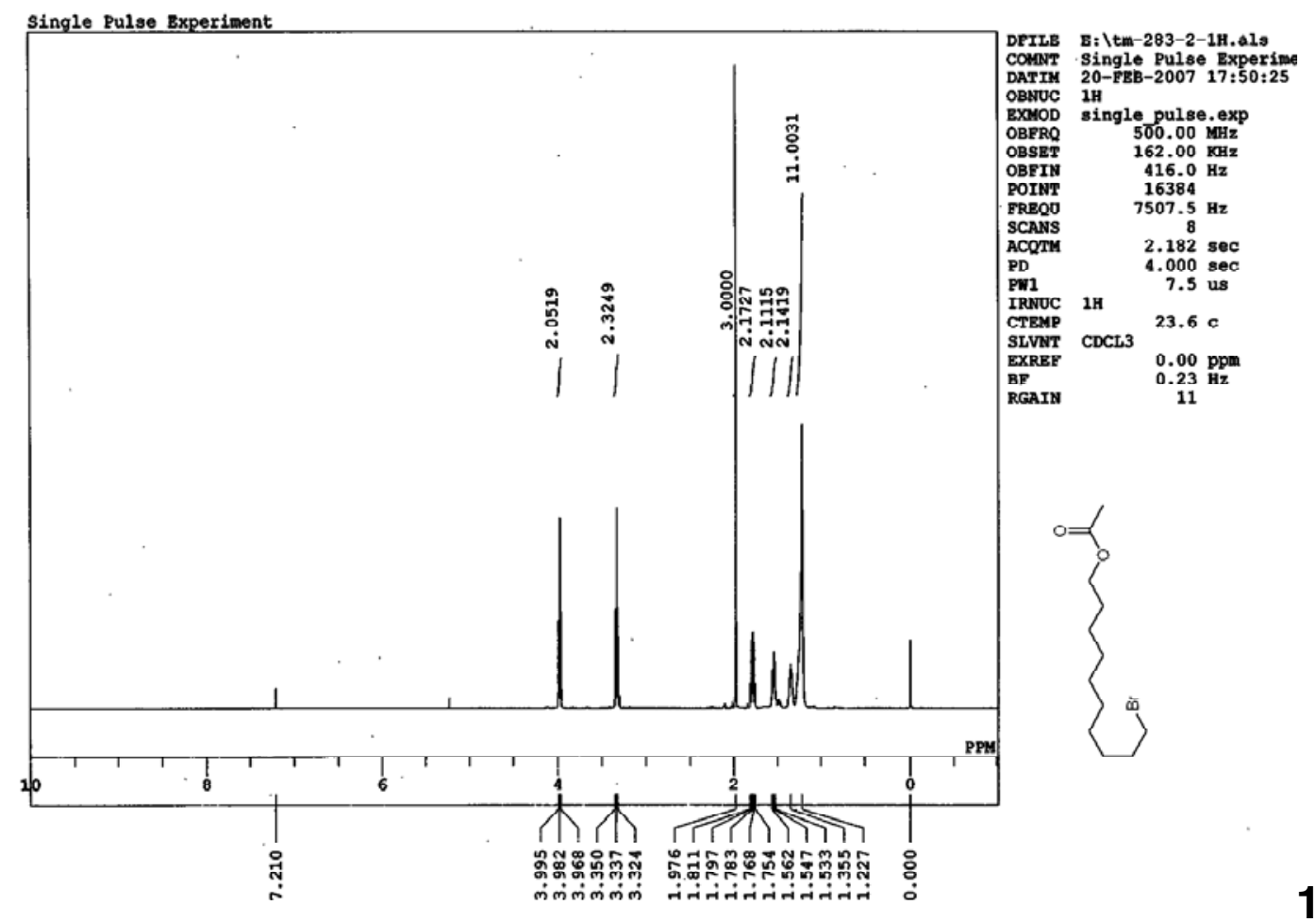

1k

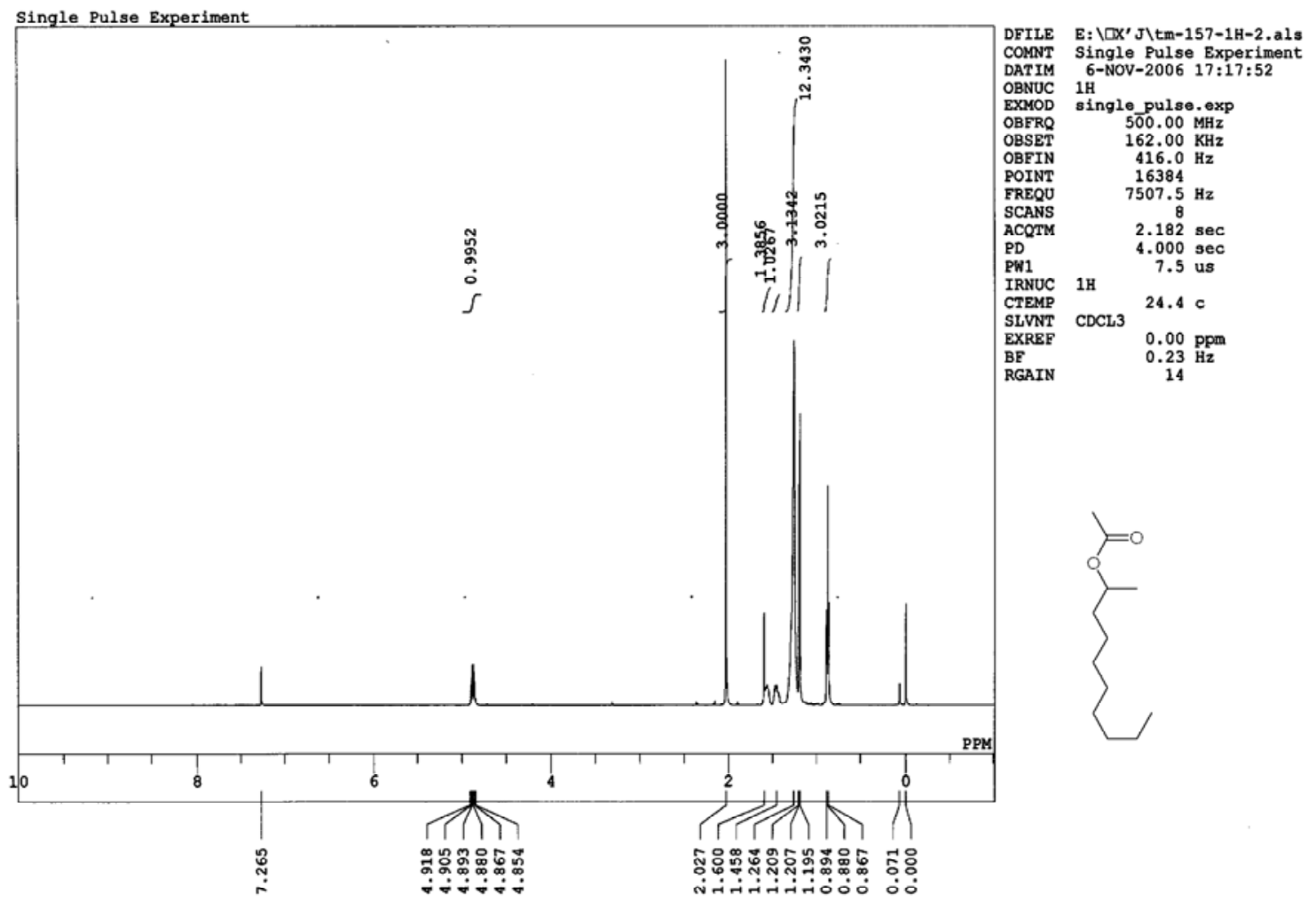



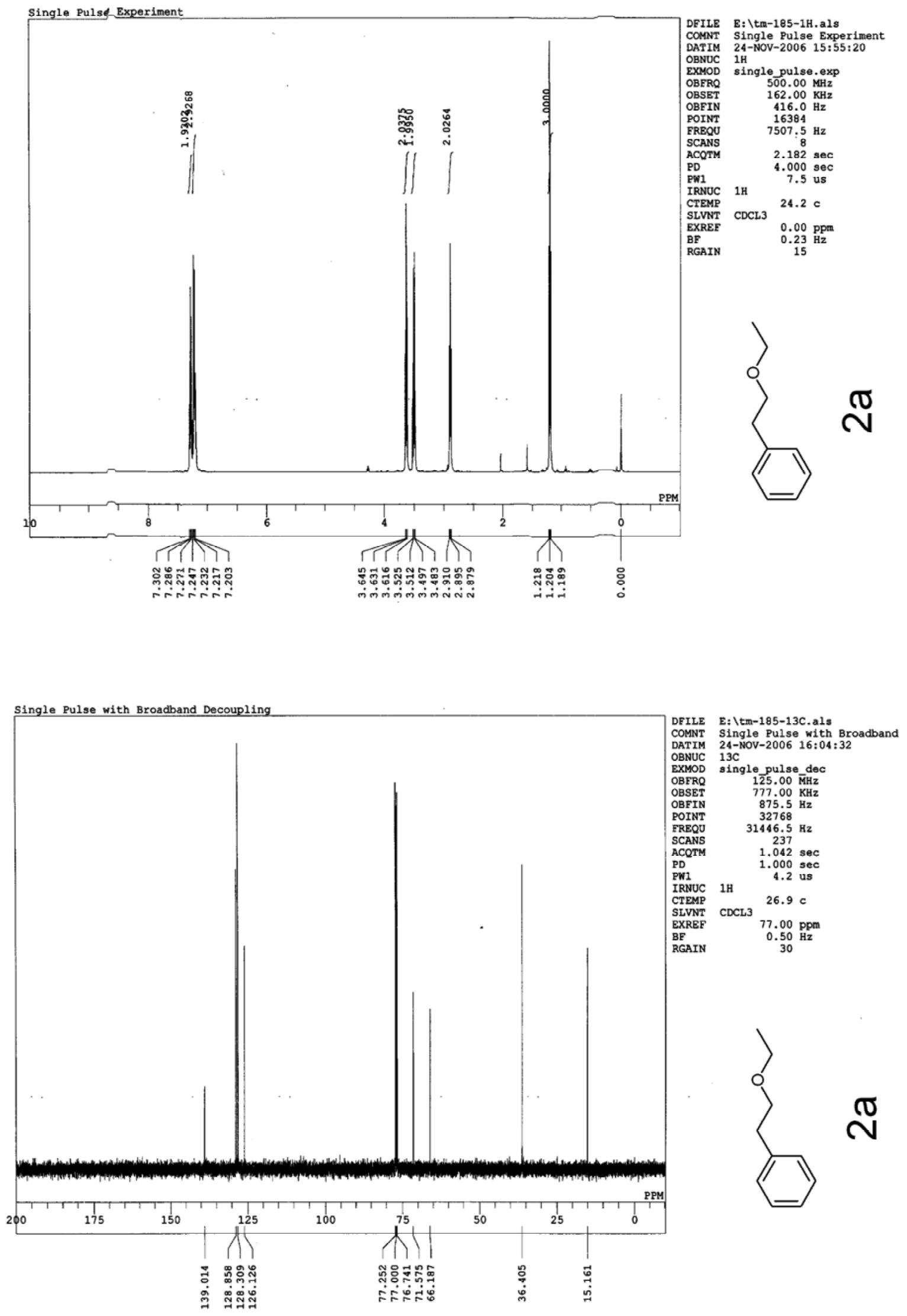

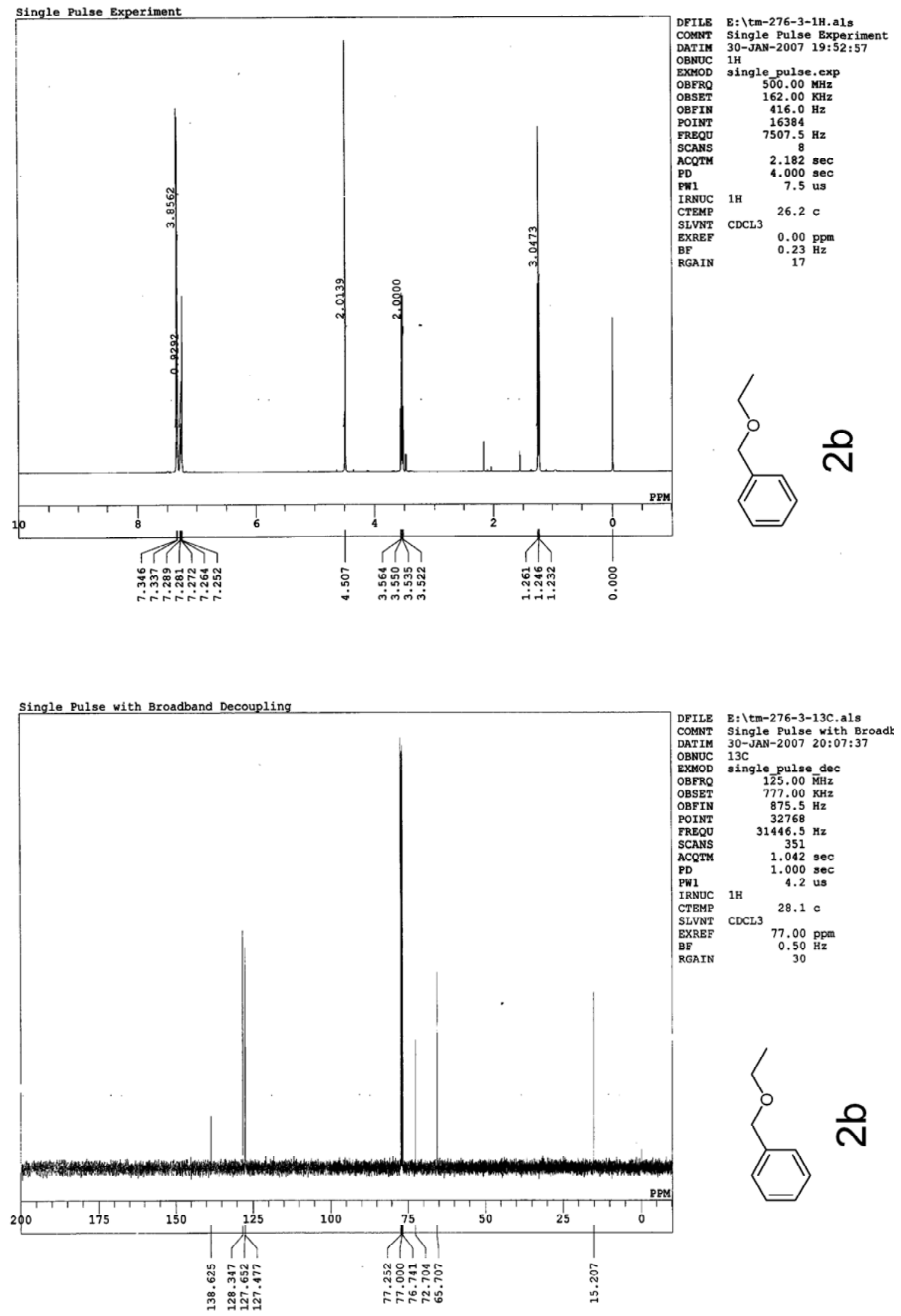

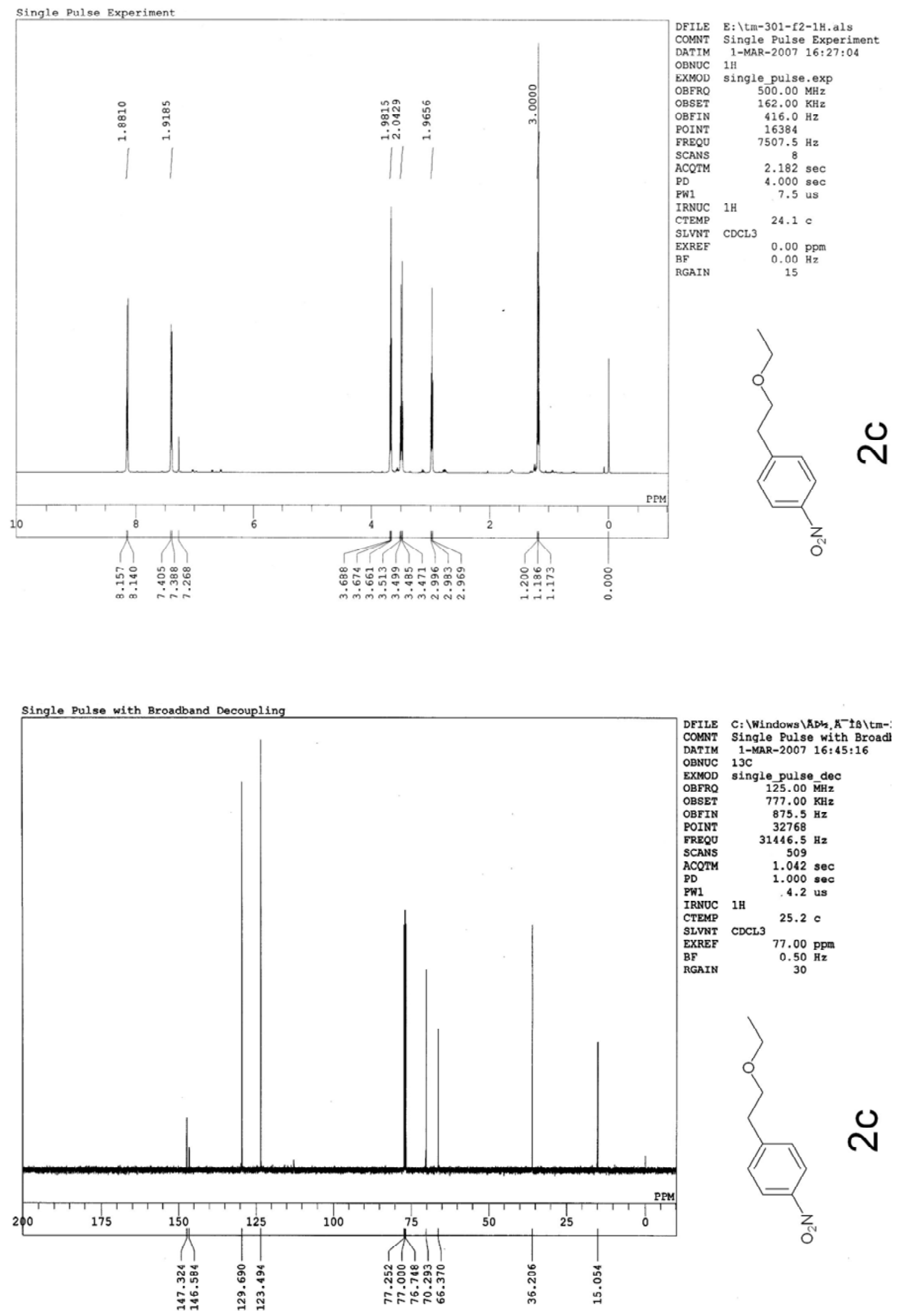

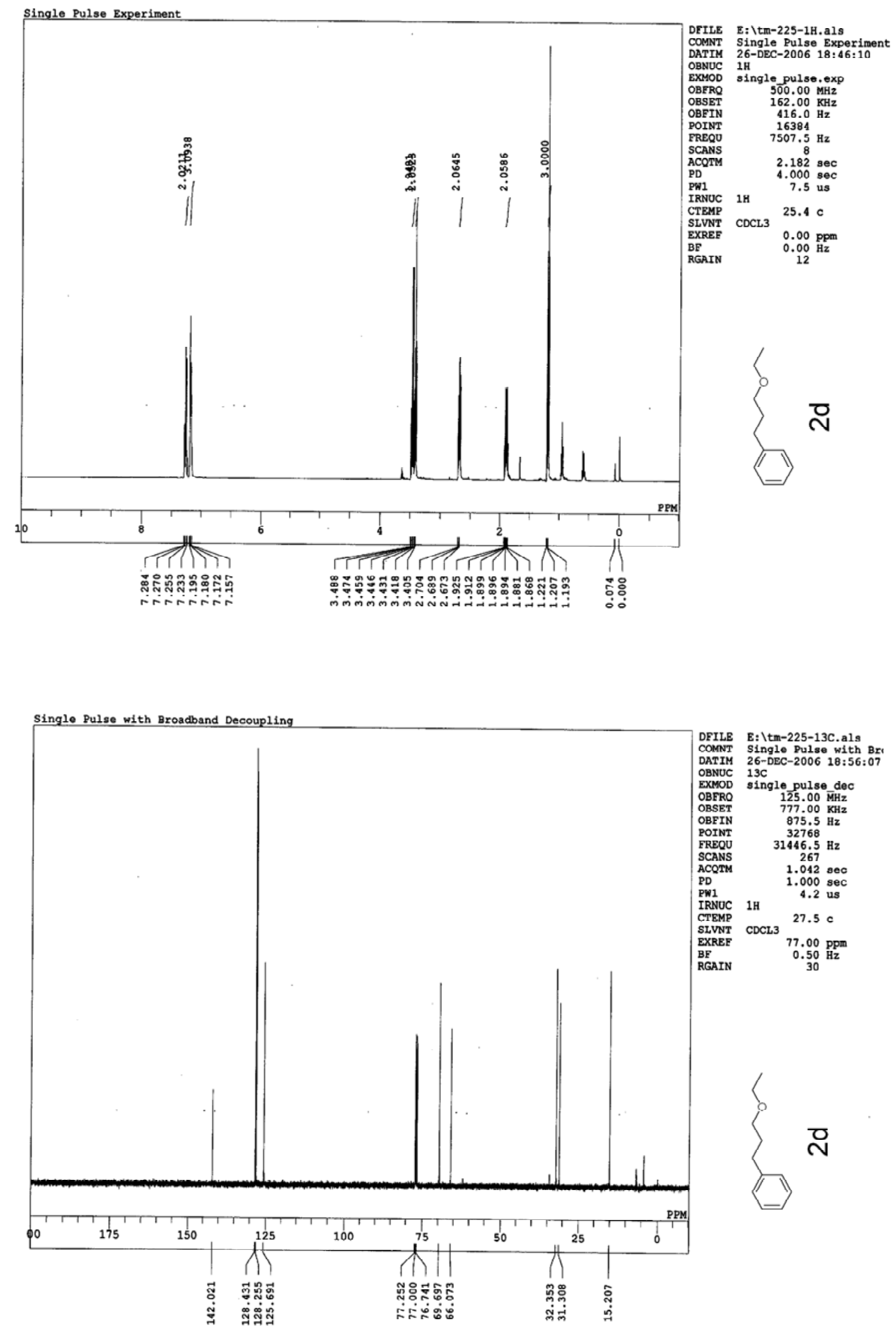

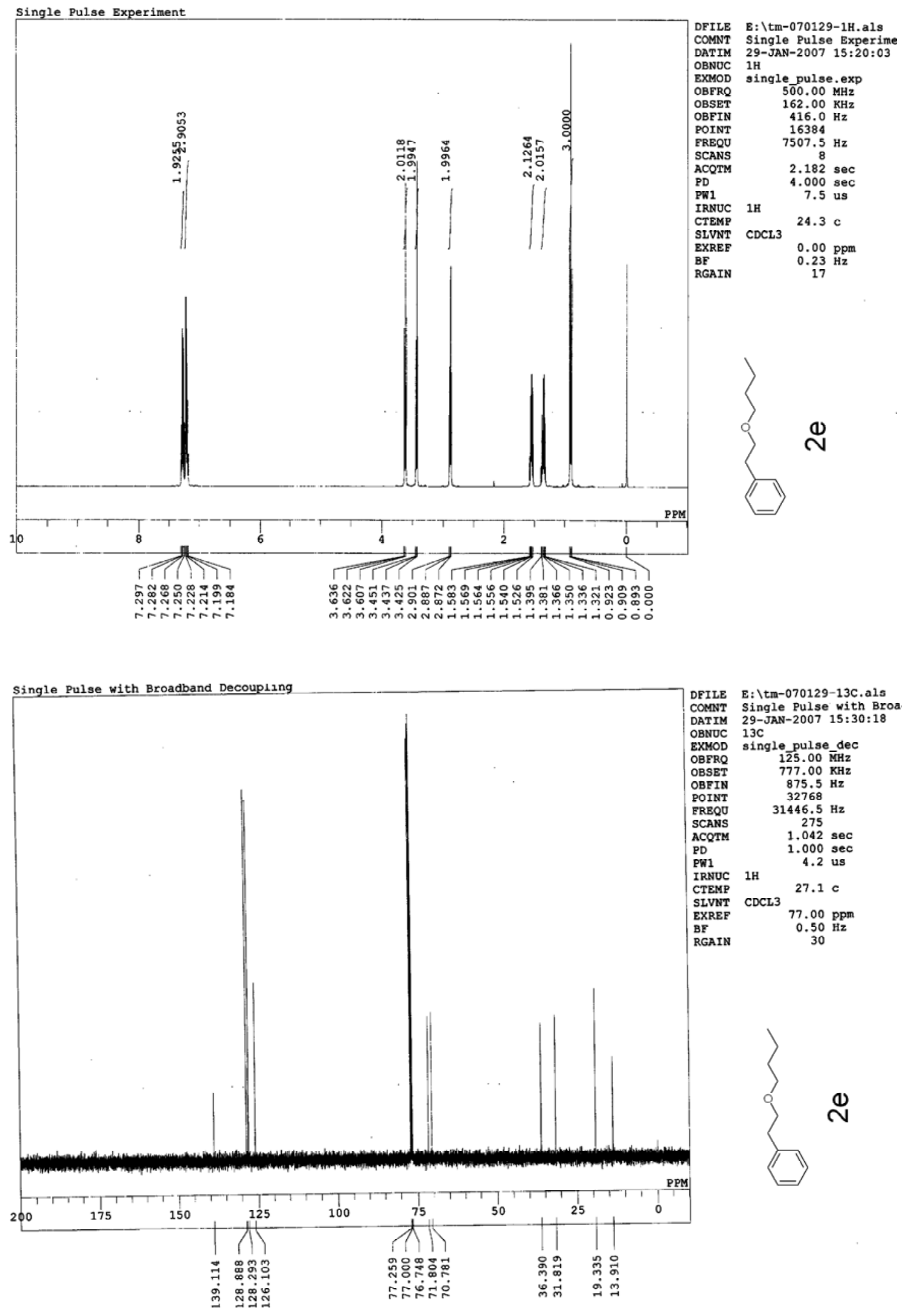

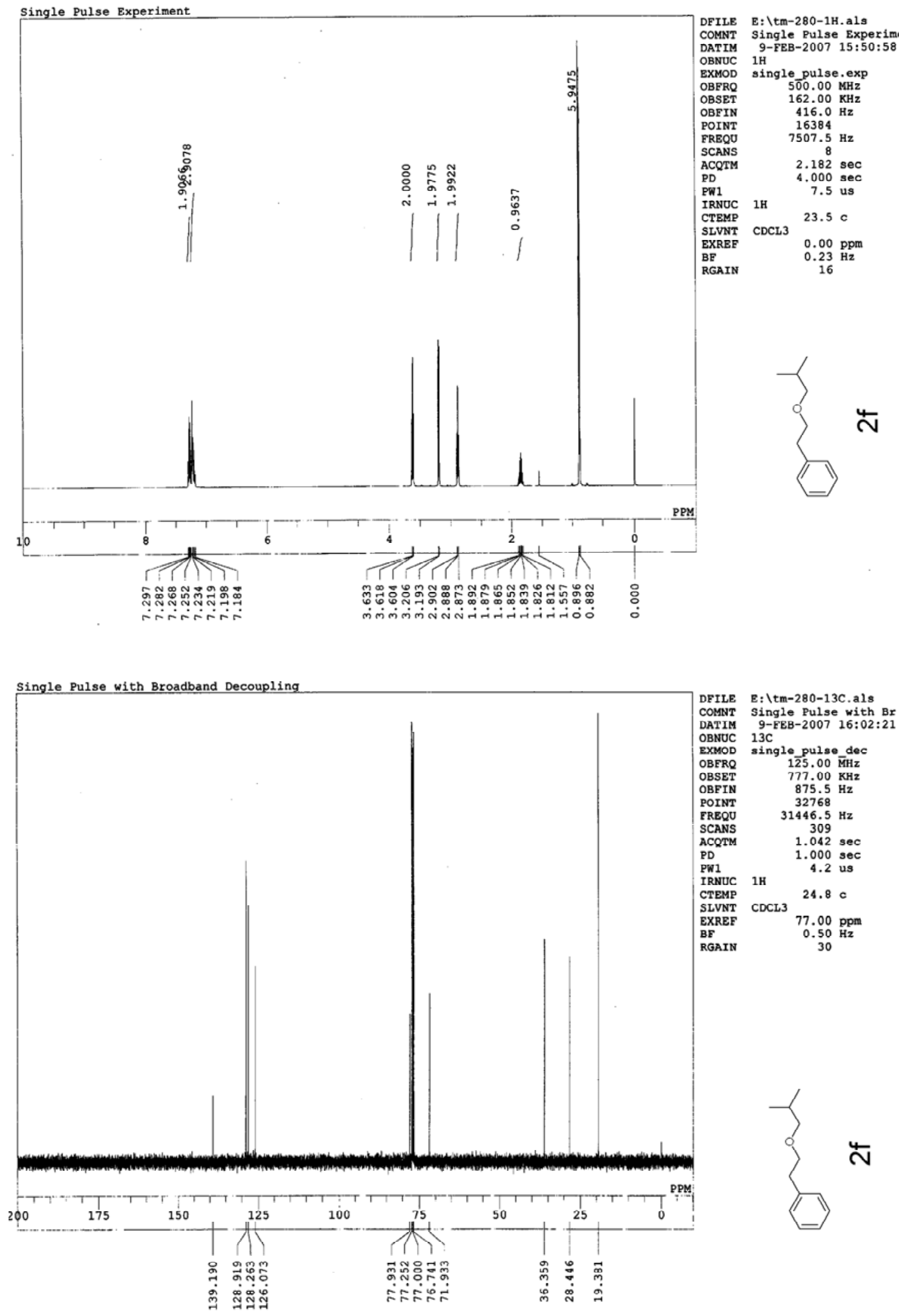

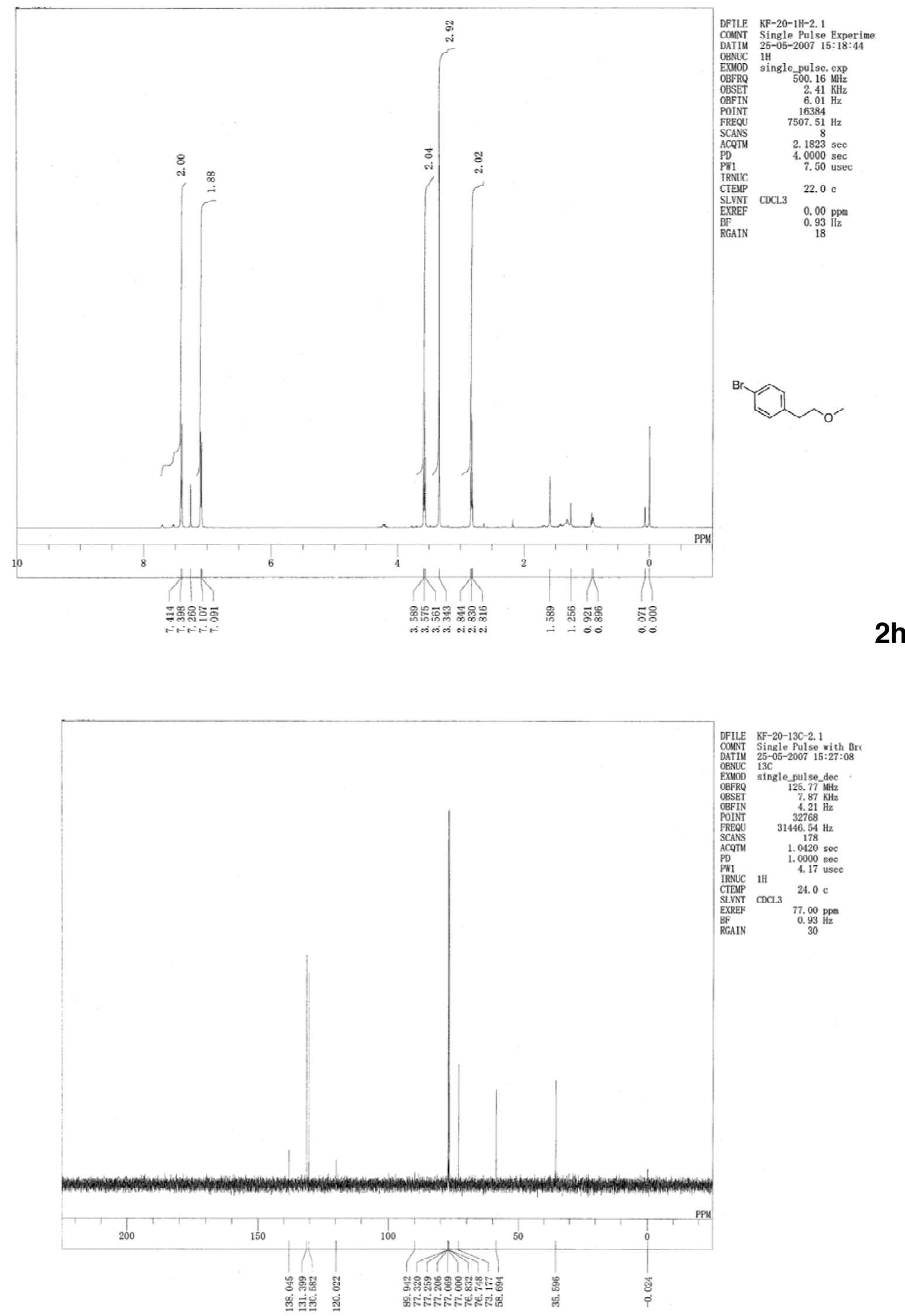

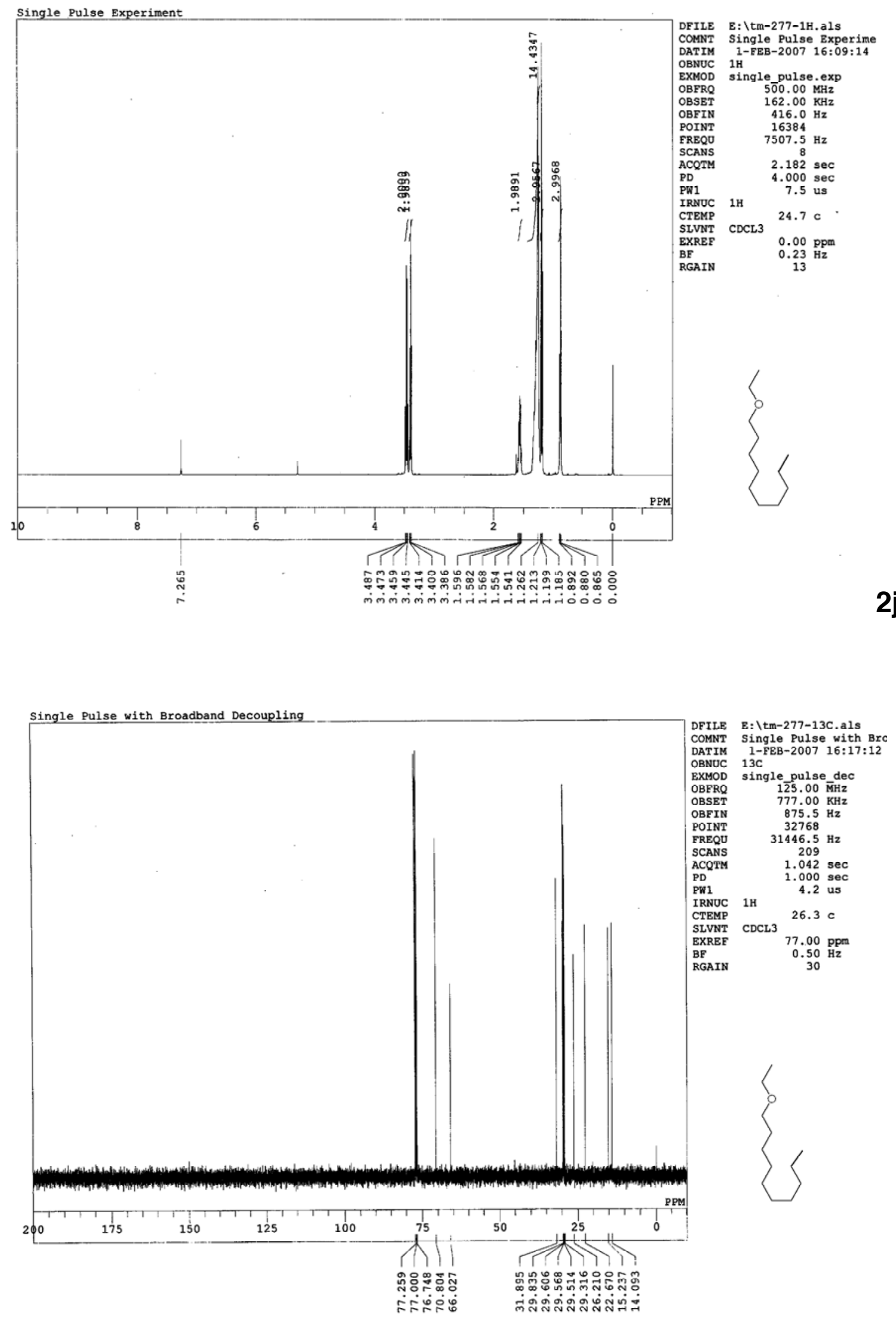

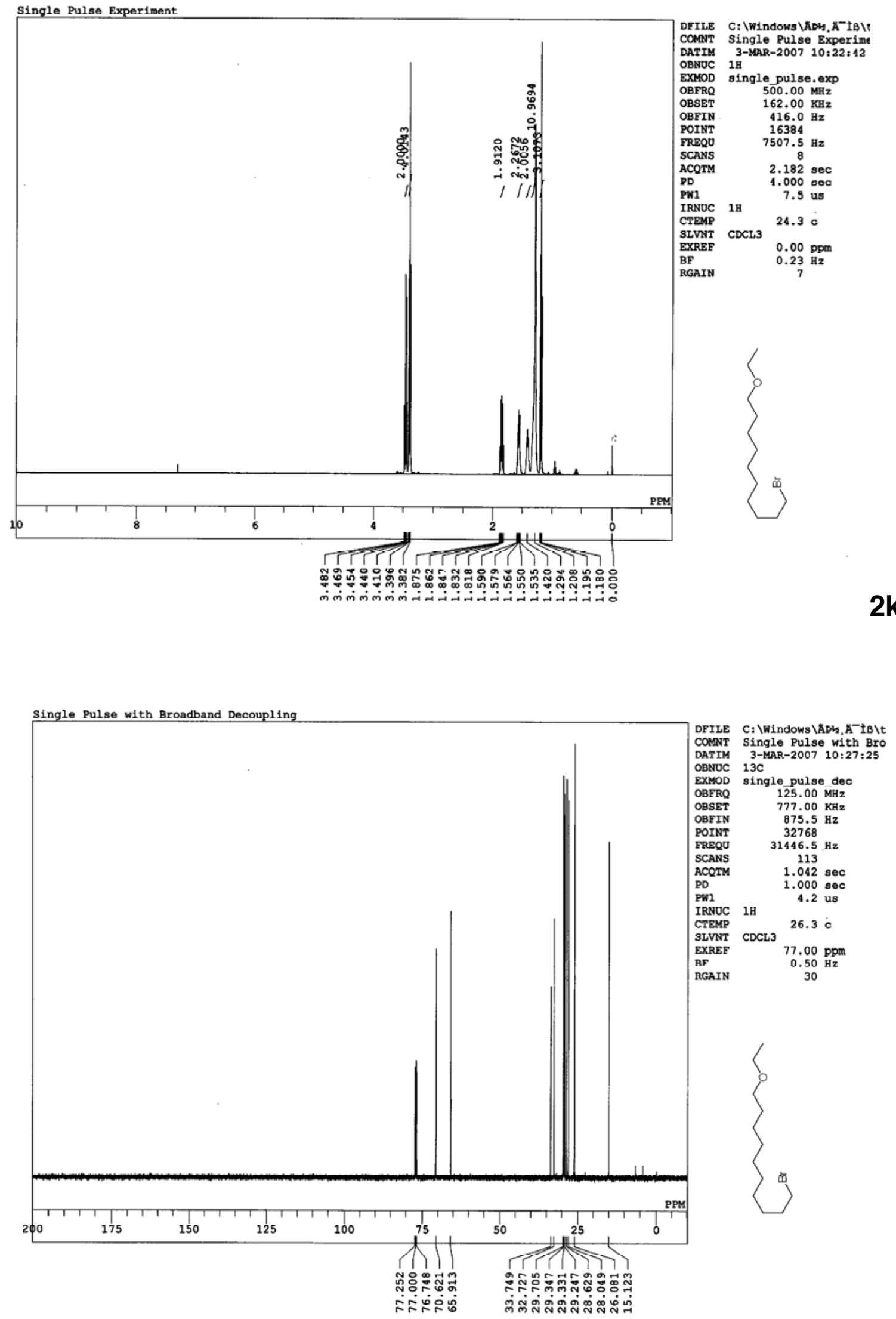

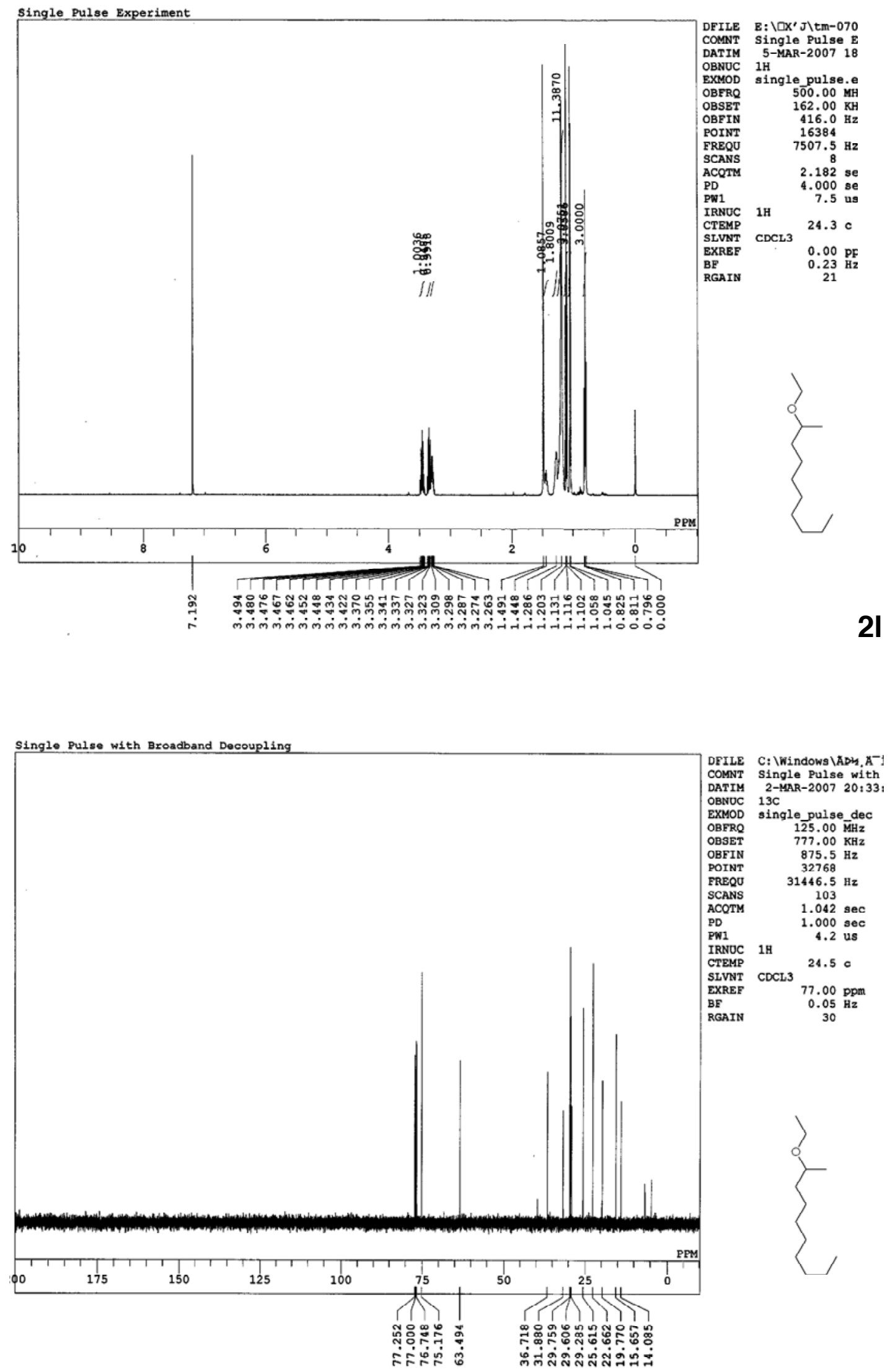

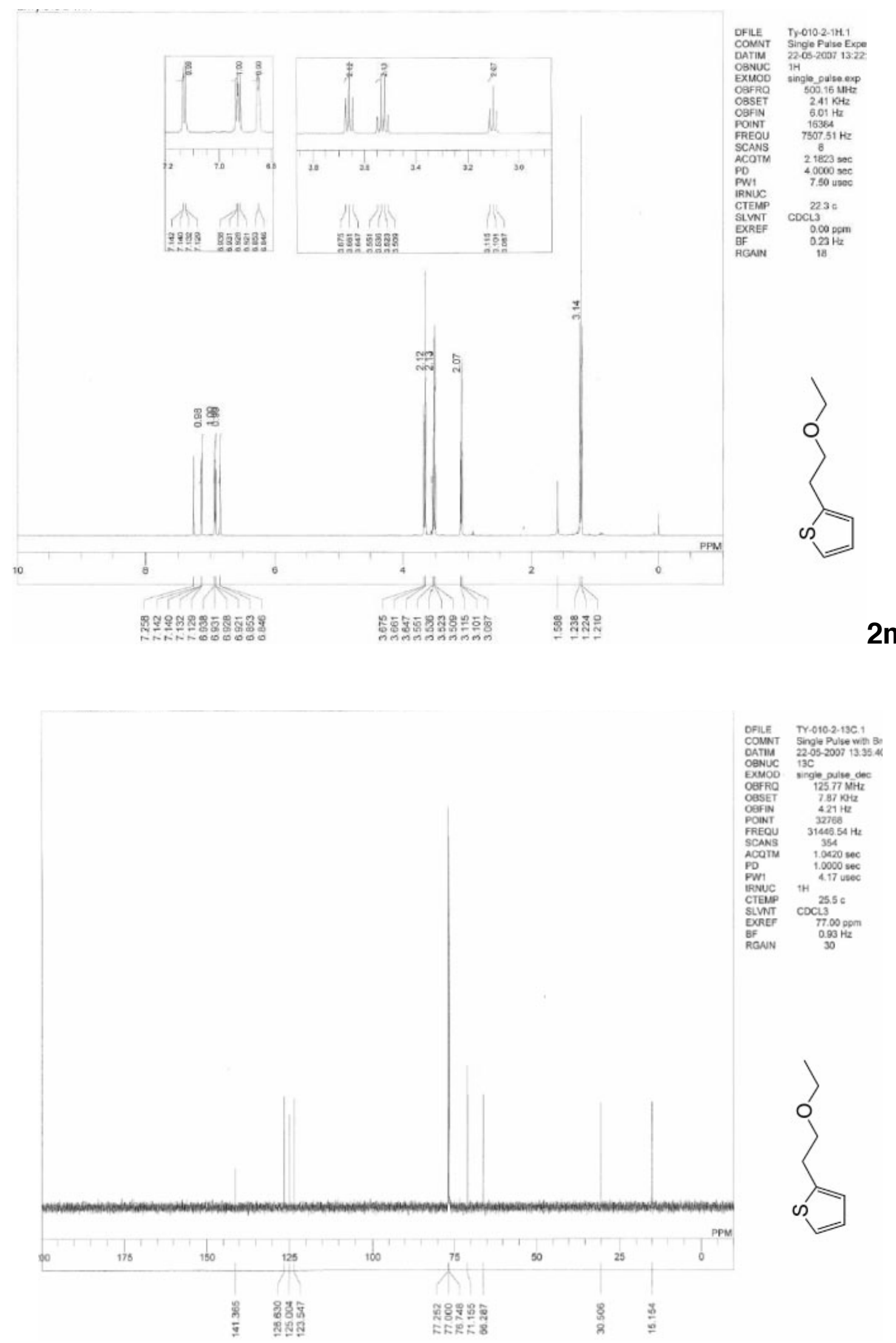

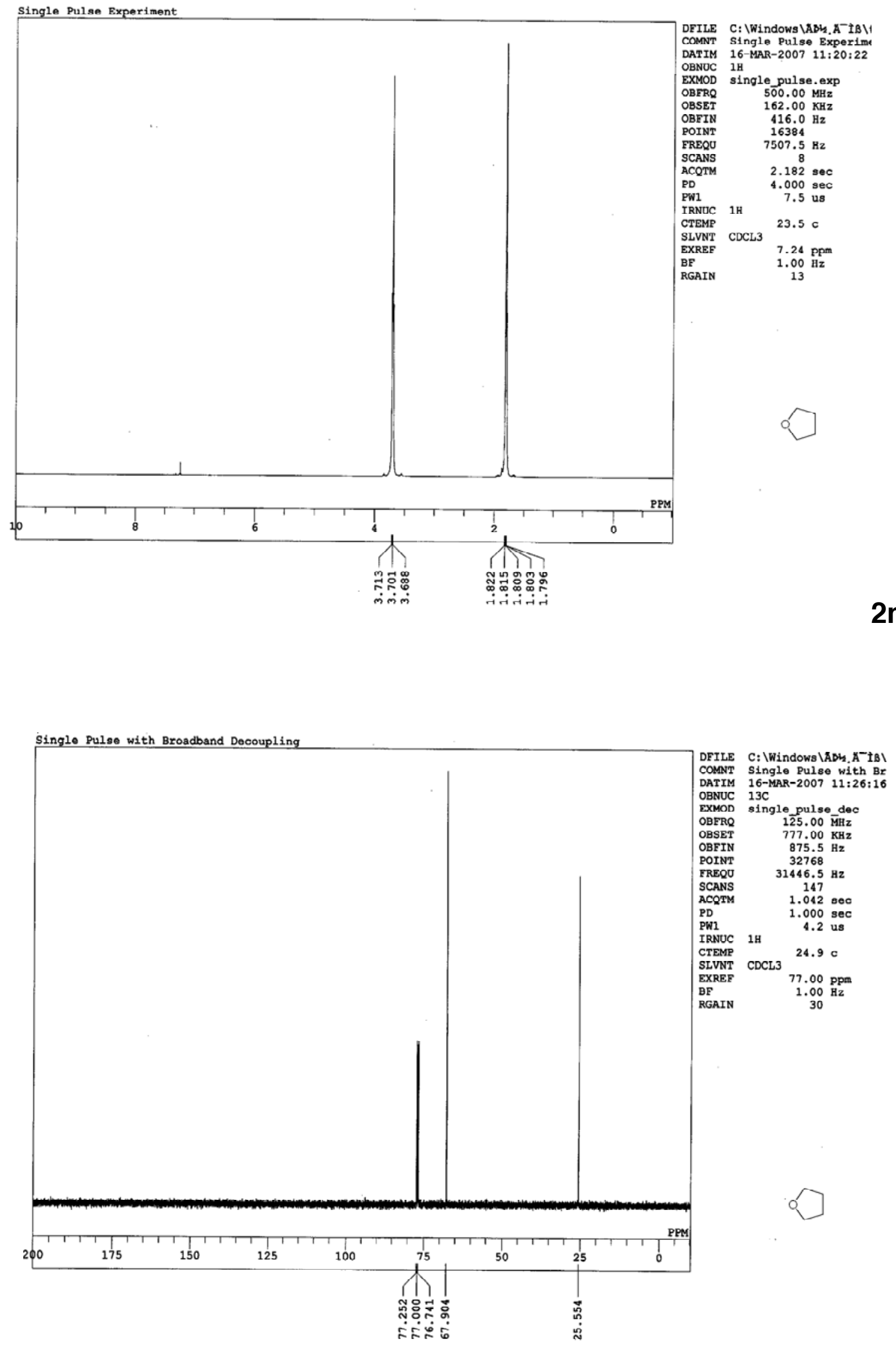

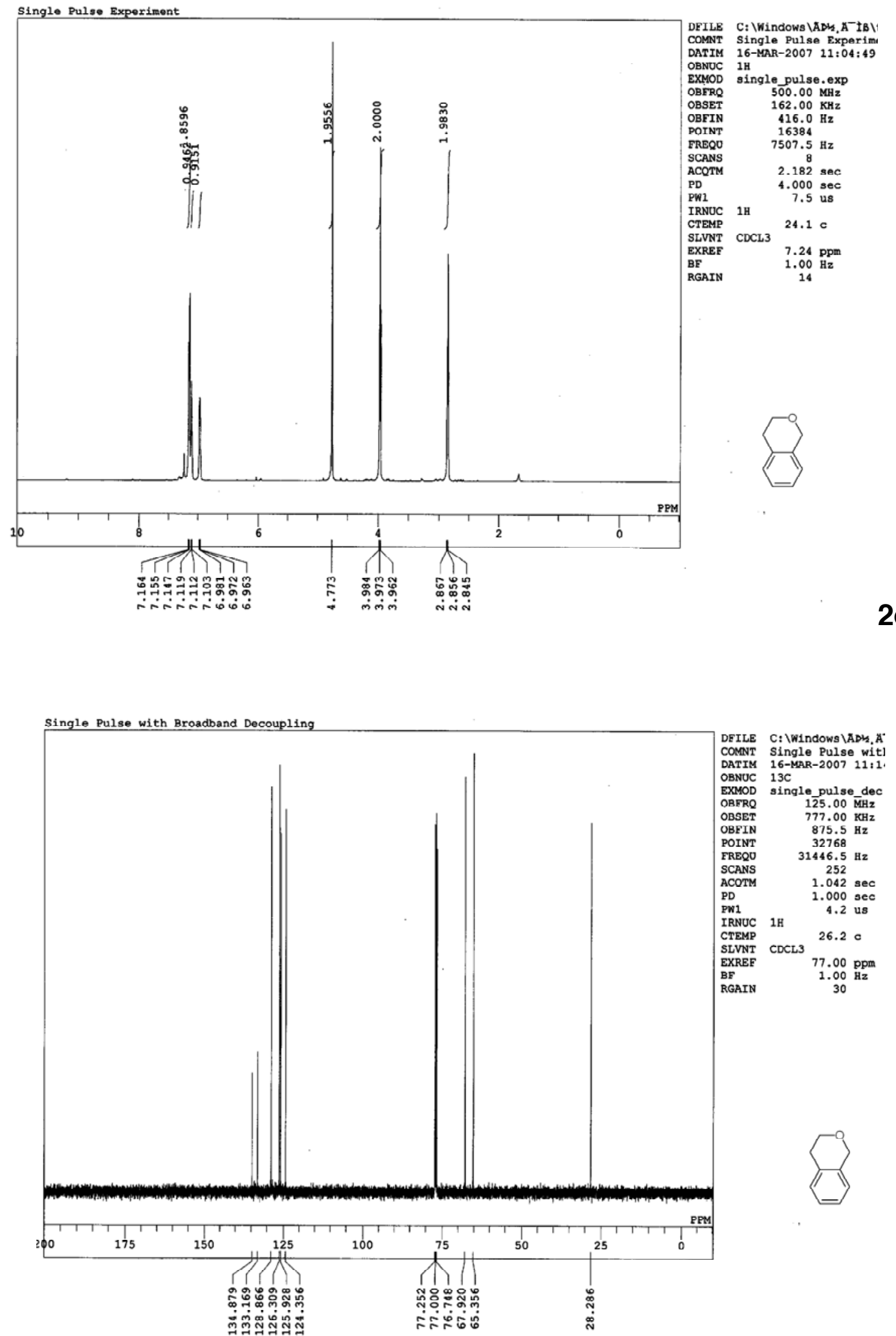

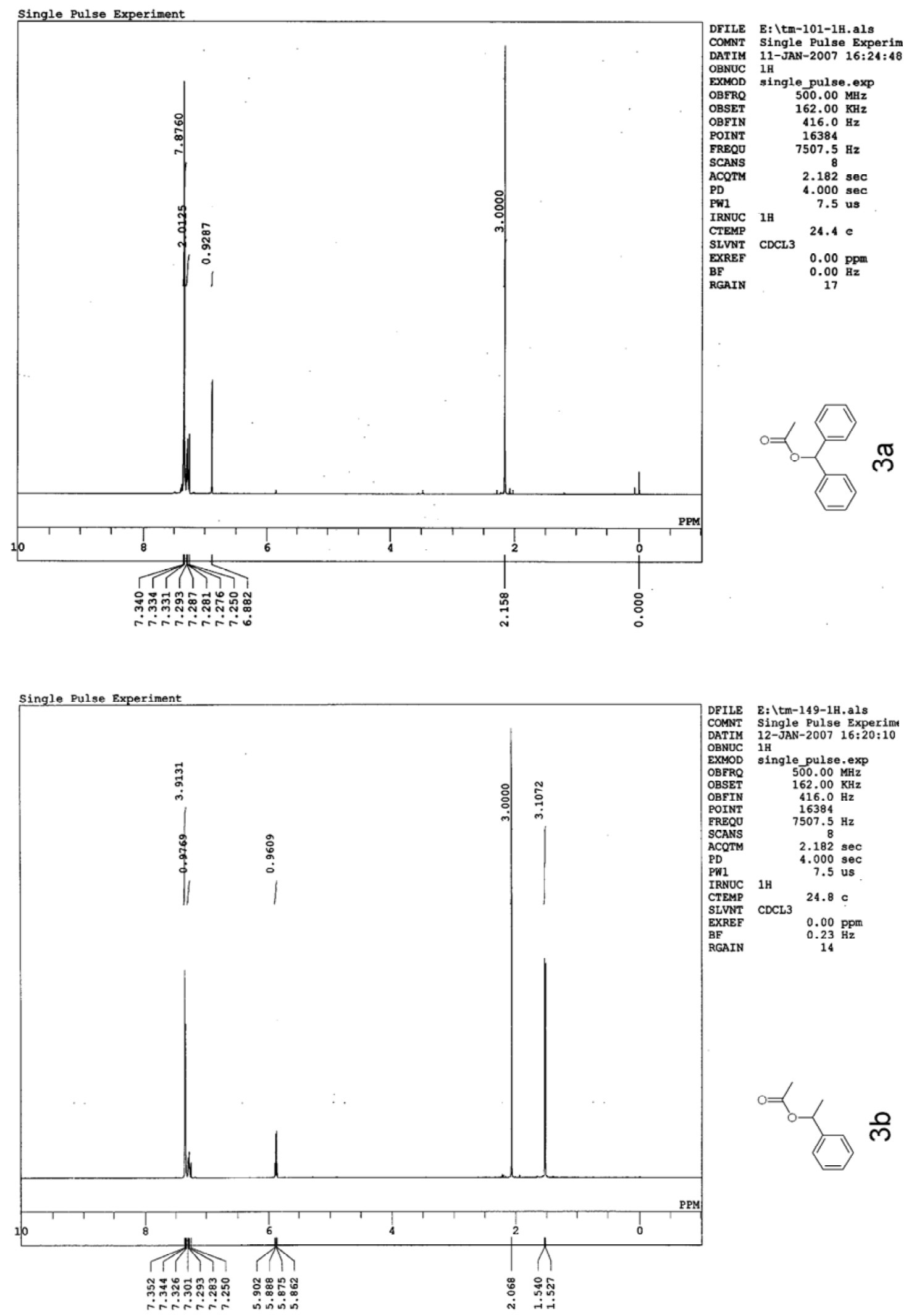

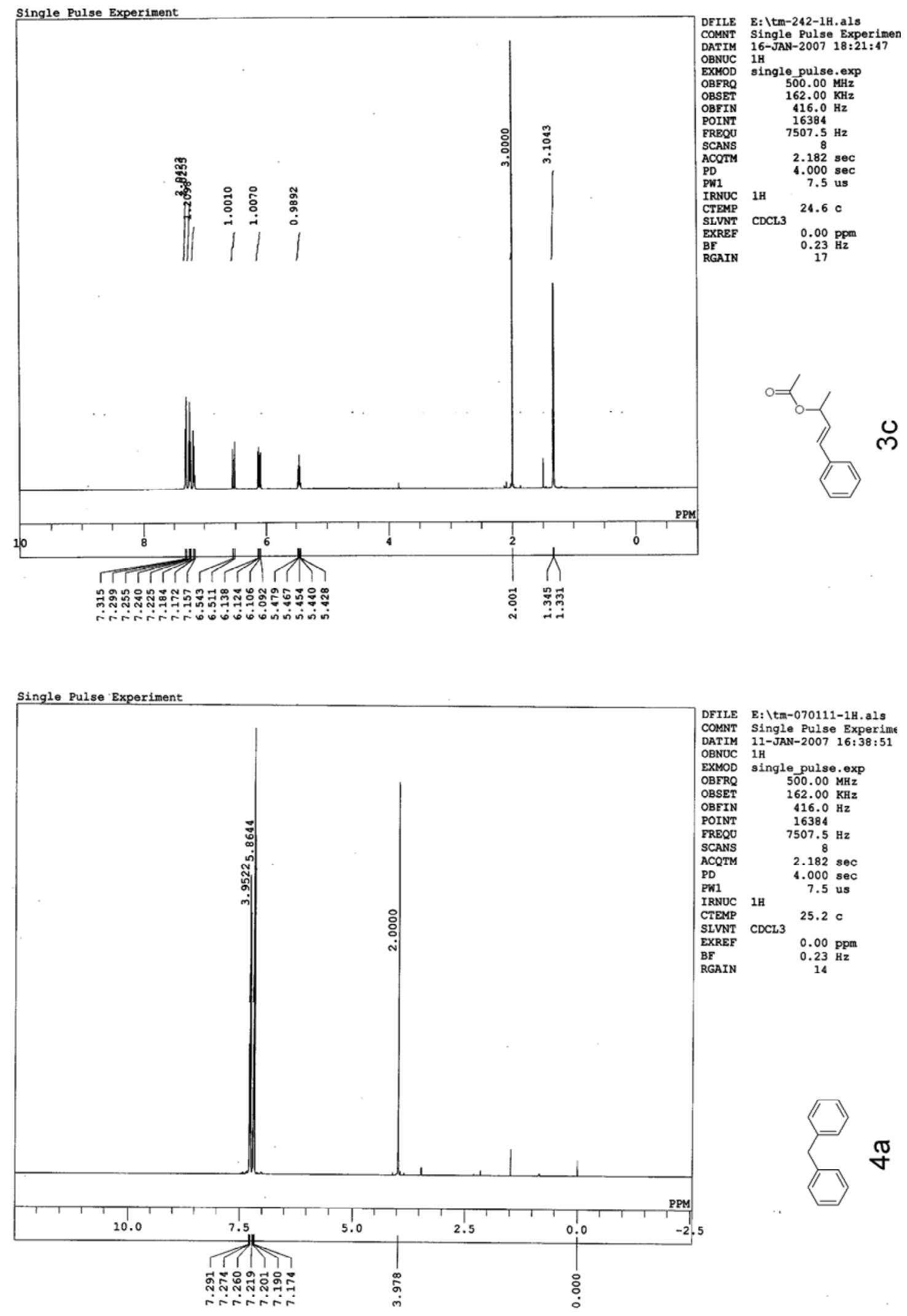

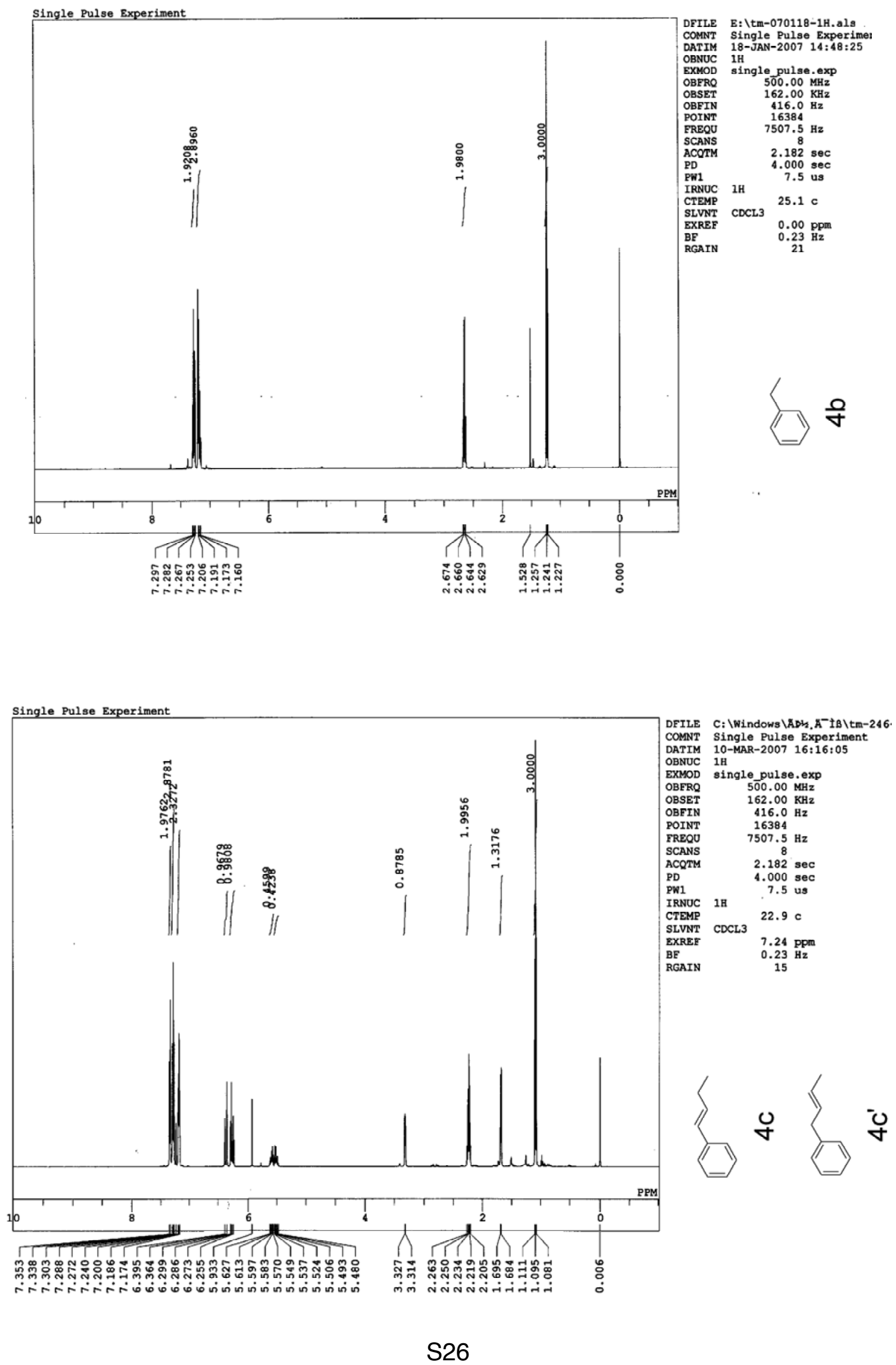

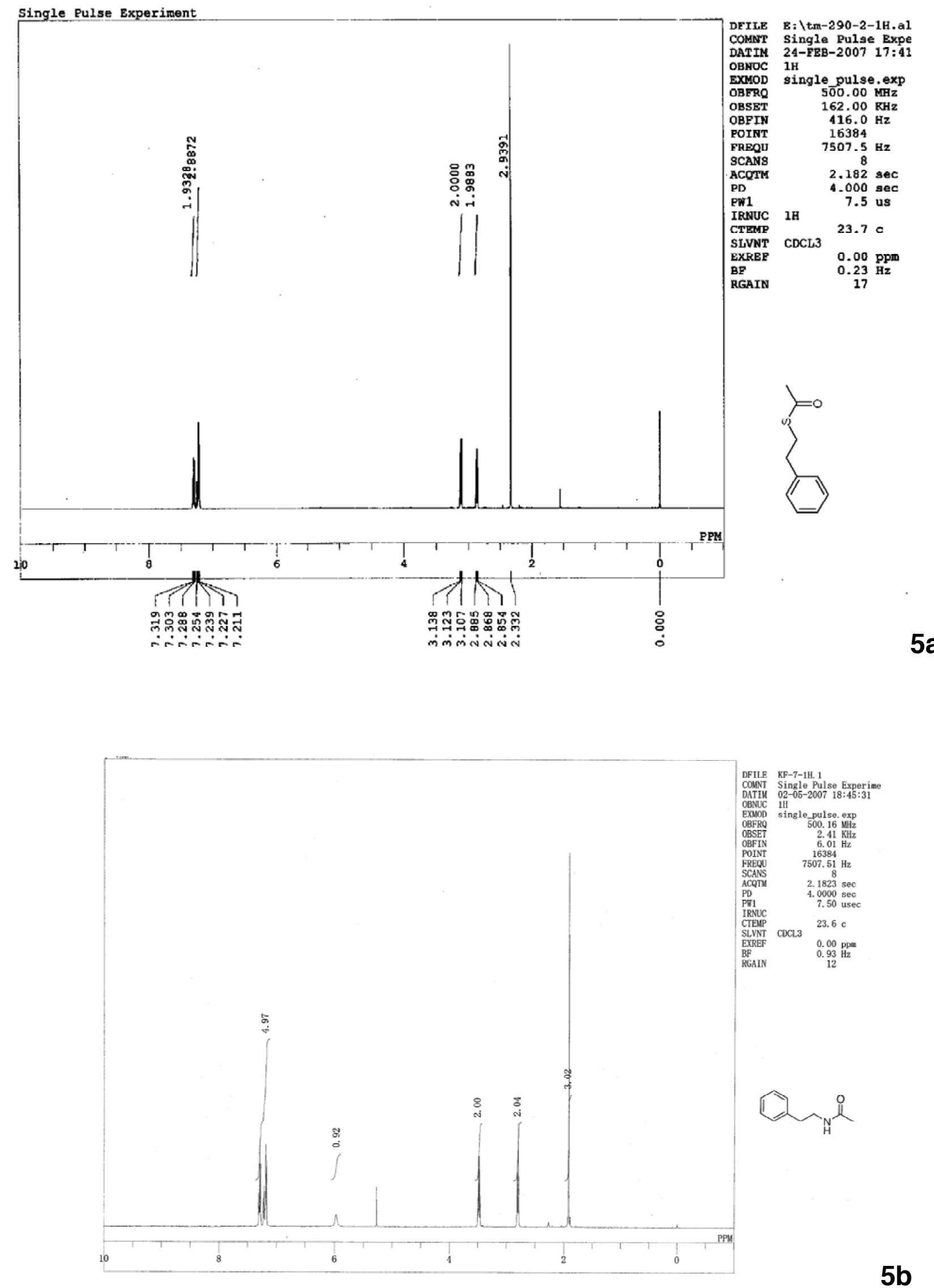

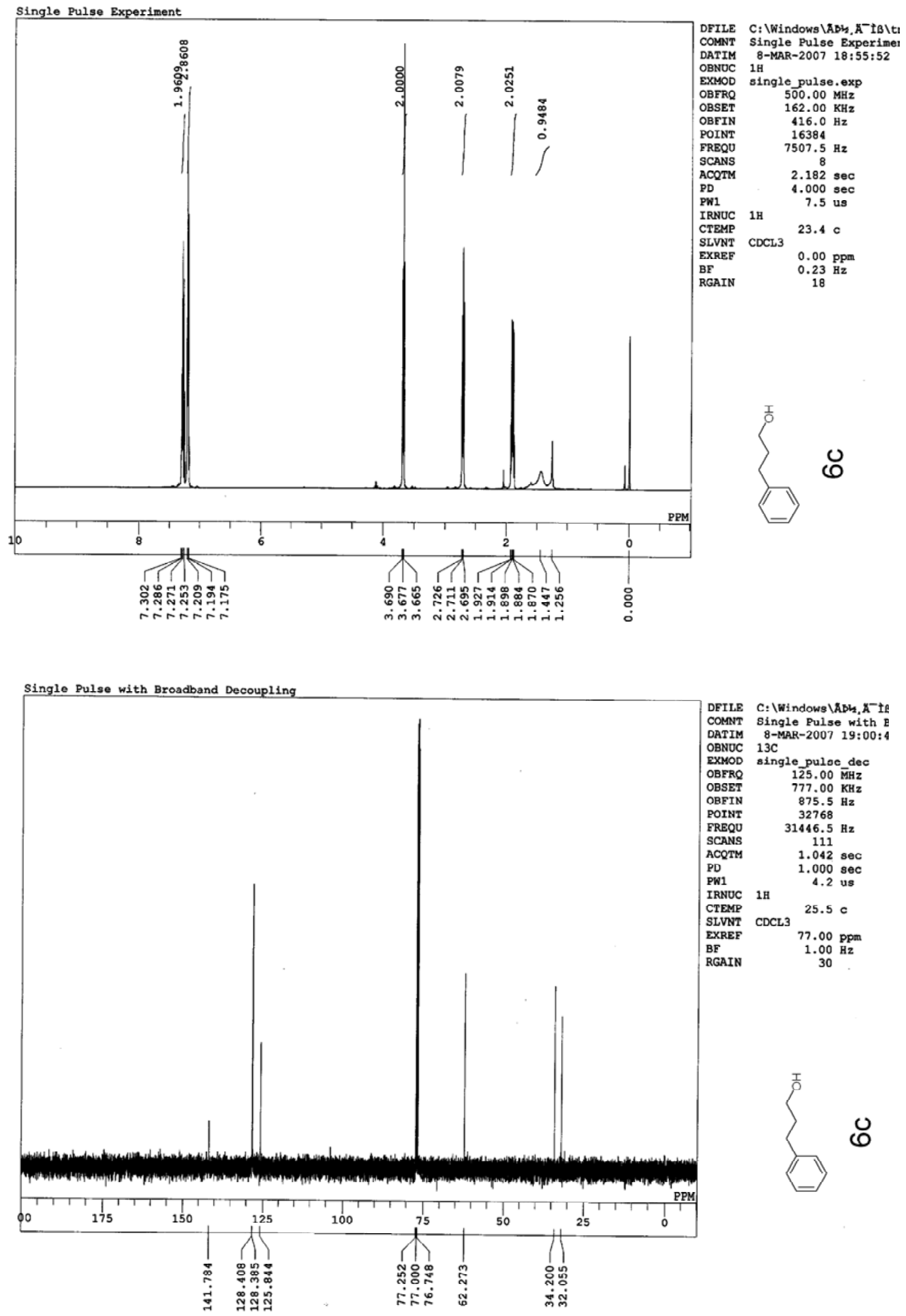

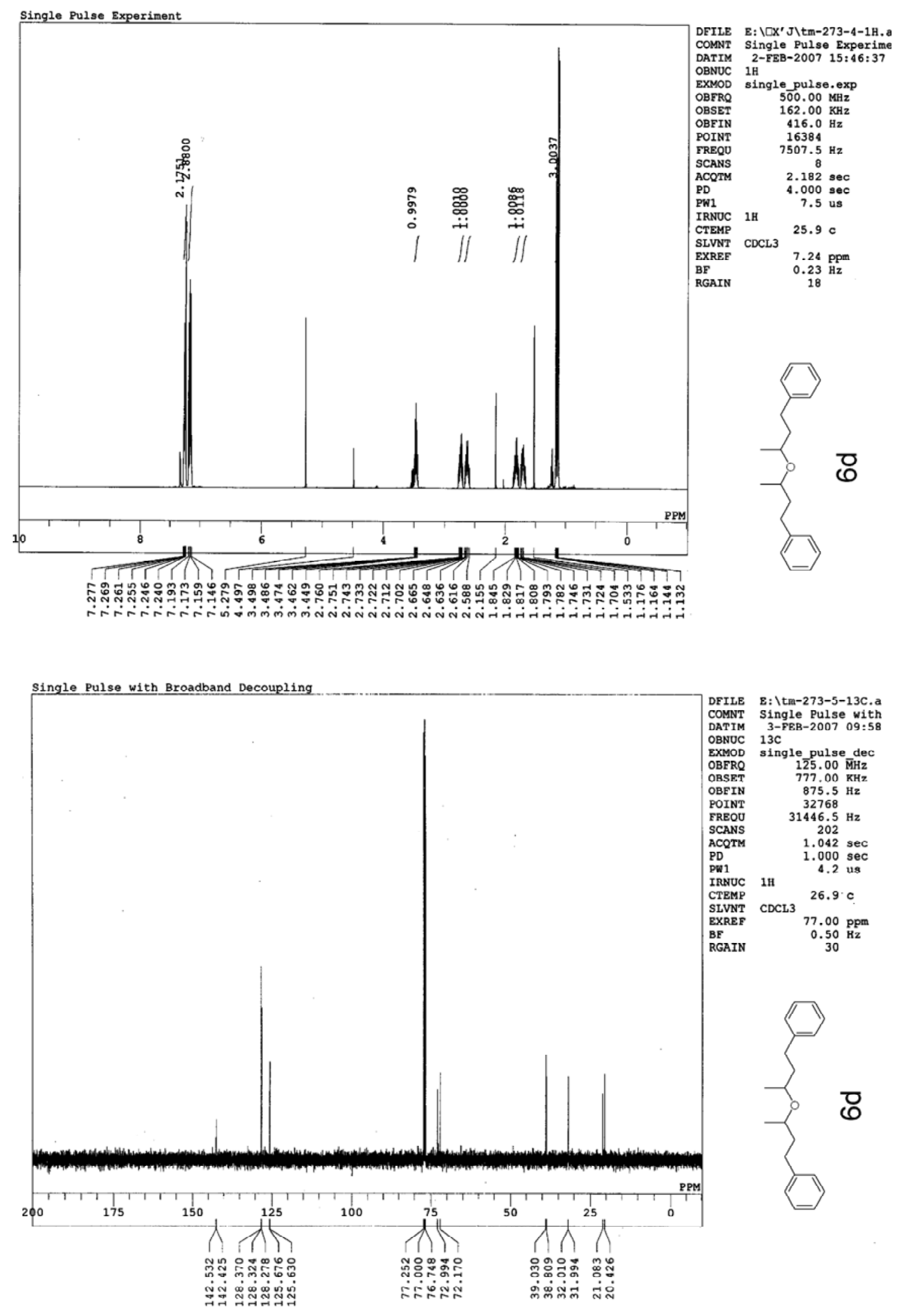